\title{
THE EVOLUTION OF SWIFT/BAT BLAZARS AND THE ORIGIN OF THE MeV BACKGROUND
}

\author{
M. Ajello ${ }^{1}$, L. Costamante ${ }^{2}$, R. M. Sambruna ${ }^{3}$, N. Gehrels ${ }^{3}$, J. Chiang $^{1}$, A. Rau ${ }^{4}$, A. Escala ${ }^{1,5}$, J. Greiner $^{6}$, J. Tueller $^{3}$, \\ J. V. WALL ${ }^{7}$, AND R. F. MUSHOTZKY ${ }^{3}$ \\ ${ }^{1}$ SLAC National Laboratory and Kavli Institute for Particle Astrophysics and Cosmology, 2575 Sand Hill Road, Menlo Park, CA 94025, USA; \\ majello@slac.stanford.edu \\ ${ }^{2}$ W. W. Hansen Experimental Physics Laboratory and Kavli Institute for Particle Astrophysics and Cosmology, Stanford University, Stanford, CA 94305, USA \\ ${ }^{3}$ Astrophysics Science Division, Mail Code 661, NASA Goddard Space Flight Center, Greenbelt, MD 20771, USA \\ ${ }^{4}$ Caltech Optical Observatories, MS 105-24, California, Institute of Technology, Pasadena, CA 91125, USA \\ ${ }^{5}$ Departamento de Astronomía, Universidad de Chile, Casilla 36-D, Santiago, Chile \\ ${ }^{6}$ Max Planck Institut für Extraterrestrische Physik, P.O. Box 1603, 85740 Garching, Germany \\ ${ }^{7}$ Physics and Astronomy Department, University of British Columbia, 6224 Agricultural Road, Vancouver V6T 1Z1, Canada \\ Received 2009 January 12; accepted 2009 May 5; published 2009 June 12
}

\begin{abstract}
We use three years of data from the Swift/Burst Alert Telescope (BAT) survey to select a complete sample of X-ray blazars above $15 \mathrm{keV}$. This sample comprises 26 flat-spectrum radio quasars (FSRQs) and $12 \mathrm{BL}$ Lacertae (BL Lac) objects detected over a redshift range of $0.03<z<4.0$. We use this sample to determine, for the first time in the $15-55 \mathrm{keV}$ band, the evolution of blazars. We find that, contrary to the Seyfert-like active galactic nuclei (AGNs) detected by BAT, the population of blazars shows strong positive evolution. This evolution is comparable to the evolution of luminous optical quasi-stellar objects (QSOs) and luminous X-rayselected AGNs. We also find evidence for an epoch dependence of the evolution as determined previously for radio-quiet AGNs. We interpret both these findings as a strong link between accretion and jet activity. In our sample, the FSRQs evolve strongly, while our best fit shows that BL Lac objects might not evolve at all. The blazar population accounts for 10\%-20\% (depending on the evolution of the BL Lac objects) of the cosmic Xray background (CXB) in the $15-55 \mathrm{keV}$ band. We find that FSRQs can explain the entire CXB emission for energies above $500 \mathrm{keV}$ solving the mystery of the generation of the $\mathrm{MeV}$ background. The evolution of luminous FSRQs shows a peak in redshift $\left(z_{c}=4.3 \pm 0.5\right)$ which is larger than the one observed in QSOs and X-rayselected AGNs. We argue that FSRQs can be used as tracers of massive elliptical galaxies in the early universe.
\end{abstract}

Key words: cosmology: observations - diffuse radiation - galaxies: active - galaxies: jets - surveys - X-rays: diffuse background

Online-only material: color figures

\section{INTRODUCTION}

Blazars constitute the most extreme class of active galactic nuclei (AGNs). Their broadband and highly variable emission is due to a relativistic jet pointing close to our line of sight (e.g., Blandford \& Rees 1978). In the framework of the AGN unified model, which ascribes the observed features of AGNs to orientation effects (Antonucci 1993; Urry \& Padovani 1995), the properties of misaligned blazars are consistent with those of radio galaxies. Indeed, the two blazar subpopulations, BL Lacertae (BL Lac) objects and flat-spectrum radio quasars (FSRQs), are thought to be the beamed counterparts of low- and high-luminosity radio galaxies, respectively (Wall \& Jackson 1997; Willott et al. 2001). Both classes of objects are normally found only in the nuclei of giant elliptical galaxies (e.g., Falomo et al. 2000; O'Dowd et al. 2002).

Blazars have been extensively studied at radio (Dunlop \& Peacock 1990; Wall et al. 2005), soft X-ray (Giommi \& Padovani 1994; Rector et al. 2000; Wolter \& Celotti 2001; Caccianiga et al. 2002; Beckmann et al. 2003; Padovani et al. 2007), and $\mathrm{GeV}$ energies (Hartman et al. 1999). It seems consolidated that FSRQs evolve positively (i.e., there were more blazars in the past; Dunlop \& Peacock 1990) up to a redshift cutoff which depends on luminosity (e.g., Padovani et al. 2007; Wall 2008). In this respect, FSRQs evolve similarly to the population of X-ray-selected, radio-quiet, AGNs (Ueda et al. 2003; Hasinger et al. 2005; La Franca et al. 2005). On the other hand, the evolution of BL Lac objects remains a matter of debate, since they were found to evolve negatively in a few cases (e.g., Rector et al. 2000; Beckmann et al. 2003) and not evolving at all in other ones (Caccianiga et al. 2002; Padovani et al. 2007).

Deriving the luminosity function of a class of objects allows us to understand the properties of the parent population and to estimate the diffuse (unresolved) background produced by the entire class. Despite all the previous studies, the lack of a sensitive all-sky hard X-ray survey has prevented, so far, to gather a sizable sample of blazars and to study their evolution in the $>10 \mathrm{keV}$ band. For these reasons, the contribution of blazars to the X-ray background ( $>10 \mathrm{keV}$ ) has never been quantified. The aim of this study is to address all these questions using data from the Swift/BAT instrument.

\subsection{The High-Energy Background}

Radio-quiet AGNs are more abundant than blazars and have been shown to be the major constituent of the cosmic X-ray background (CXB; Ueda et al. 2003; Treister \& Urry 2005; Gilli et al. 2007). This consolidates the idea that the CXB emission is the result of accretion onto supermassive black holes. More precisely, the X-ray emission of AGNs is due to Compton up-scatter of UV photons (generated in the inner part of the accretion disk) by high-energy electrons which populate a region above the disk commonly referred to as corona. This process, known as Comptonization, was first proposed by Zdziarski (1986). The bulk of the electron population present in the corona is 
expected to be thermal and this naturally produces a cutoff in the spectrum of AGNs. The detection of the AGN cutoffs, detected at energies between $50 \mathrm{keV}$ and $400 \mathrm{keV}$ by OSSE (Madejski et al. 1995; Zdziarski et al. 2000, and references therein) and the nondetection of Seyfert-like AGNs by EGRET (Lin et al. 1993; Dermer \& Gehrels 1995) confirms this interpretation.

Population synthesis models normally assume that all emission-line AGNs have a cutoff in the 200-500 keV energy range (e.g., Ueda et al. 2003; Gilli et al. 2007,). The effect of the cutoff combined with the cosmic evolution of AGNs implies that the contribution of radio-quiet AGNs to the CXB emission above $\sim 200 \mathrm{keV}$ is negligible. Thus, the high-energy CXB emission in the 200-10,000 keV energy range remains currently unexplained. A few candidates have been proposed to explain this background. One is the $\gamma$-ray emission originated from nuclear decays from Type Ia supernovae (SNe Ia; Clayton \& Ward 1975; Zdziarski 1996; Watanabe et al. 1999). However, on the basis of measurements of the cosmic SN Ia rates, recent studies showed that the background flux expected from SNe Ia is about an order of magnitude lower than the observed CXB emission (Ahn et al. 2005; Strigari et al. 2005). Annihilation of dark matter particles has also been discussed, but no viable light (with "MeV" mass) dark matter particle candidate has been found (Ahn \& Komatsu 2005, and references therein).

Very recently, Inoue et al. (2008) discussed the possibility that the hot corona may contain a small fraction (relative to the whole population) of nonthermal electrons. These electrons might be powered by magnetic reconnections in a similar way as it happens during solar flares (e.g., Shibata et al. 1995). In this framework, a faint nonthermal component present in millions of AGNs might explain the observed background. However, due to the lack of sensitive instruments surveying the MeV sky, this hypothetical nonthermal emission of AGNs has never been detected.

Blazars, whose emission extend from the radio to the $\mathrm{TeV}$ band, are certainly contributing, despite their relative low space density, to the high-energy background (both in the X-ray and $\gamma$-ray energy bands). In particular, an important role is certainly played by the so-called "MeV blazars" whose inverse Compton (IC) peak is located in the MeV band (Bloemen et al. 1995; Sikora et al. 2002; Sambruna et al. 2006). An attempt to quantify the contribution of blazars to the high-energy background has been performed by Giommi et al. (2006) using a multifrequency-selected sample of blazars. Despite the uncertainties related to the extrapolation from the microwave to the hard $\mathrm{X}$ ray energy band, they conclude that blazars can explain $\sim 10 \%$ of the CXB emission in the $2-10 \mathrm{keV}$ energy band and possibly $100 \%$ of the background above $500 \mathrm{keV}$.

In this work, we use a complete sample of blazars detected in the Swift/BAT survey to derive, for the first time at these energies, the X-ray luminosity function (XLF) and the cosmic evolution of blazars and to assess their contribution to the high-energy background. The paper is organized as follows. In Section 2, we describe how the blazar sample was selected among the BAT extragalactic sources and discuss its incompleteness. In Section 3, we introduce the maximum likelihood method which is used to determine the blazar evolution. The luminosity function of the BAT blazars is derived in Section 4, and in Section 5 it is used to quantify the contribution of blazars to the diffuse background. We discuss the results of our analysis in Section 7. Throughout this paper, we assume a standard concordance cosmology $\left(H_{0}=70 \mathrm{~km} \mathrm{~s}^{-1} \mathrm{Mpc}^{-1}, \Omega_{M}=1-\Omega_{\Lambda}=0.3\right)$.

\section{THE SWIFT/BAT SAMPLE}

The Burst Alert Telescope (BAT; Barthelmy et al. 2005) onboard the Swift satellite (Gehrels et al. 2004), represents a major improvement in sensitivity for imaging of the hard Xray sky. BAT is a coded mask telescope with a wide field of view (FOV; $120^{\circ} \times 90^{\circ}$ partially coded) aperture sensitive in the $15-200 \mathrm{keV}$ domain. The main goal of BAT is to locate gammaray bursts (GRBs). While chasing new GRBs, BAT surveys the hard X-ray sky with an unprecedented sensitivity. Thanks to its wide FOV and its pointing strategy, BAT monitors continuously up to $80 \%$ of the sky every day. Thanks to this quasi-random pointing strategy and the large FOV, BAT exposure is uniform on the whole sky.

Results of the BAT survey (Markwardt et al. 2005; Ajello et al. 2008a; Tueller et al. 2009) show that BAT reaches a sensitivity of $\sim 1 \mathrm{mCrab}^{8}$ in $1 \mathrm{Ms}$ of exposure. Given its sensitivity and the large exposure already accumulated in the whole sky, BAT poses itself as an excellent instrument for studying populations whose emission is faint in hard X-rays.

For the analysis presented here, we used Swift/BAT survey observations performed between 2005 March and 2008 March. Data screening and processing was performed according to the recipes presented in Ajello et al. (2008a). The chosen energy interval is $15-55 \mathrm{keV}$. The lower limit is dictated by the energy threshold of the detectors. The upper limit was chosen as to avoid the presence of strong background lines which could worsen the overall sensitivity (see Ajello et al. 2008b, for details about the BAT background). The all-sky image is obtained as the weighted average of all the shorter observations. The average exposure time in our image is $4.3 \mathrm{Ms}$, being $2.0 \mathrm{Ms}$ and $6.8 \mathrm{Ms}$ the minimum and maximum exposure times, respectively. The final image shows a Gaussian normal noise and we identified source candidates as excesses above the $5 \sigma$ level. All the candidates are then fitted with the BAT point-spread function (using the standard BAT tool batcelldetect) to derive the best source position. Moreover, in order to avoid problems related to source confusion and sample incompleteness, we considered only sources at high $\left(|b|>15^{\circ}\right)$ Galactic latitude. This analysis is based on mean source fluxes determined over the three year period spanned by the survey. Our high-latitude sample comprises 305 sources. Of these 40 are Galactic sources (mainly $\mathrm{X}$-ray binaries) and six are galaxy clusters (already comprised in the sample of Ajello et al. 2009). The remainder are 247 extragalactic sources and 12 unidentified objects. The exact composition of the sample is reported in Table 1 .

Being the BAT survey not a flux-limited survey, but rather a significance-limited one, it is important to address how the survey flux limit changes over the sky area. This is often referred to as sky coverage, that is the distribution of the survey's area as a function of limiting flux. Its knowledge is very important when performing population studies as the ones described in the following sections. The reader is referred to Ajello et al. (2008a) for how to derive the sky coverage which as a function of the minimum detectable flux $F_{\min }$ is defined as the sum of the area covered to fluxes $f_{i}<F_{\min }$ :

$$
\Omega\left(<F_{\min }\right)=\sum_{i}^{N} A_{i} \quad f_{i}<F_{\min },
$$

where $N$ is the number of image pixels and $A_{i}$ is the area associated with each of them. A visual representation of the

\footnotetext{
$81 \mathrm{mCrab}$ in the $15-55 \mathrm{keV}$ band corresponds to $1.27 \times 10^{-11} \mathrm{erg} \mathrm{cm}^{-2} \mathrm{~s}^{-1}$.
} 


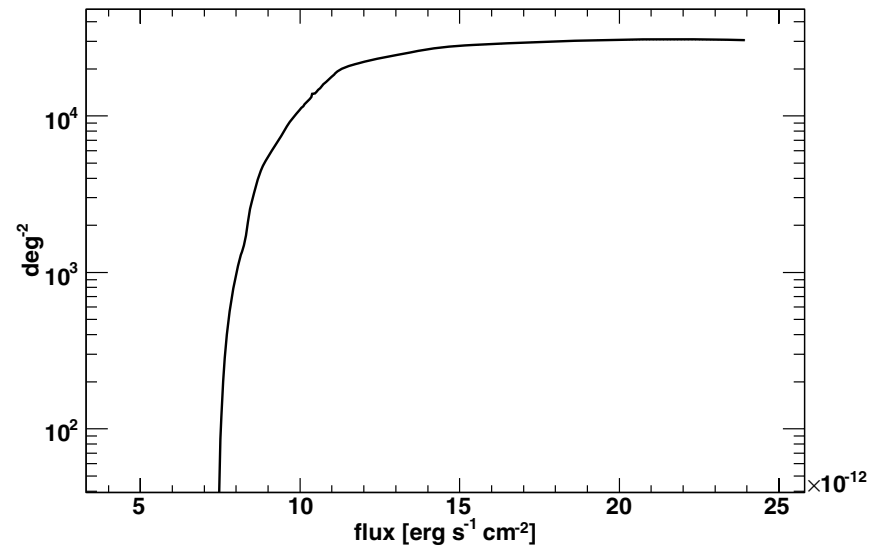

Figure 1. Sky coverage of the BAT survey as a function of minimum detectable flux for $\mathrm{S} / \mathrm{N} \geqslant 5 \sigma$ and $|b| \geqslant 15^{\circ}$ in the $15-55 \mathrm{keV}$ band.

sky coverage is reported in Figure 1 which shows clearly the good sensitivity of BAT which reaches, in our analysis (15$55 \mathrm{keV})$, a limiting sensitivity of $\sim 0.6 \mathrm{mCrab}\left(7.3 \times 10^{-12} \mathrm{erg}\right.$ $\left.\mathrm{cm}^{-2} \mathrm{~s}^{-1}\right)$.

\subsection{The Blazar Sample}

Selection of blazars in the BAT energy band is not problematic. Thanks to the deep, although sparse, coverage of the sky in soft (0.1-2.5 keV) and medium (2-10 keV) X-rays, most of the BAT sources are already well studied objects. The location accuracy of BAT was recently characterized by Tueller et al. (2009) using the all-sky sample of sources detected in 22 months of observations. They find that the $96 \%$ error radius for a $5 \sigma, 10 \sigma$, and $20 \sigma$ source is, respectively, 7..5, 3.34, and 1.59. For the identification of most of the BAT sources, we used the ROSAT All-Sky Survey Bright Source Catalog (Voges et al. 1999), the Third IBIS Catalog (Bird et al. 2007), the BAT catalogs of Markwardt et al. (2005) and Tueller et al. (2008, 2009) as well as SIMBAD and NED. We remark that all the identifications reported in the aforementioned catalogs are based on a thorough study, both in the optical and X-rays, of the properties of the BAT counterparts (e.g., Ajello et al. 2008c; Winter et al. 2008). Some 50 BAT sources, for which the above catalogs did not provide an identification, have a publicly available Swift/XRT observation. These observations provided a secure identification of the BAT objects and the results of all these followups will be discussed elsewhere (see Table 1 for a breakdown of the total sample). As shown in Ajello et al. (2008c) and Winter et al. (2008), most of the BAT AGNs show complex Xray spectra (with the presence of iron line, Compton reflection, and soft excesses) and often very large absorption $\left(N_{\mathrm{H}} \sim 10^{23}\right.$ atoms $\mathrm{cm}^{-2}$ ) which securely associates the BAT object with a nonblazar AGN (e.g., Seyfert).

In order to securely identify and classify BAT sources as blazars, we relied mainly on the blazar catalog (BZCAT) of Massaro et al. (2009) which contains only bona fide blazars. These are sources which show a dominant, broadband, nonthermal component associated with a (relativistic) jet, a variable nuclear activity, and are detected at least in radio, optical, and X-rays. Given the fact that the BZCAT does not cover the entire sky, we also used the CRATES catalog of FSRQs (see also below; Healey et al. 2007) and when necessary individual source publications. The sample of BAT blazars is reported in Table 2 along with the main properties of the sources (e.g., fluxes, signal-to-noise ratios, etc.), while the sample of Seyferts
Table 1

Composition of the BAT High-latitude Sample $\left(|b|>15^{\circ}\right.$ and S/N $\left.>5\right)$

\begin{tabular}{lc}
\hline \hline \multicolumn{1}{c}{ CLASS } & No. of Objects \\
\hline Total & 305 \\
Seyferts & 199 \\
Blazars & 38 \\
Galaxies & $4^{\mathrm{a}}$ \\
Galaxy Clusters & 6 \\
Radio Galaxies & 6 \\
Galactic Sources & $40^{\mathrm{b}}$ \\
Unidentified & 12 \\
\hline
\end{tabular}

Notes.

a These objects are candidate radio-quiet AGNs (Seyferts), identified by means of a $2-10 \mathrm{keV}$ follow-up observation, for which an optical spectrum, and thus redshift, is not yet available.

${ }^{b}$ It includes all objects of Galactic nature (i.e., pulsars, X-ray binaries, etc.).

and radio galaxies detected by BAT is reported for reference in the Appendix. For this analysis, BL Lac objects are identified as objects in which the equivalent width of the strongest emission line is less than $5 \AA$ and the optical spectrum shows a Ca II H/K break <0.4 (e.g., Urry \& Padovani 1995; Marcha et al. 1996). The blazar sample comprises 26 FSRQs and 12 BL Lac objects and the full details of the classification (including the references for it) are given in Table 2. Among the BL Lac objects, nine are of the high-frequency peaked (HBL) type, while the rest are of the low-frequency peaked (LBL) type. Figure 2 (both panels) shows that the BL Lac objects detected by BAT have a softer spectrum with respect to the FSRQ population. The average photon index of the BL Lac objects, in the BAT band is $2.5 \pm 0.5$, while the one for FSRQs is $1.6 \pm 0.3$. This is however not surprising, indeed it confirms the expectation that BAT samples the synchrotron component in BL Lac objects and the IC component in FSRQs (e.g., see the BeppoSAX results of Donato et al. 2005). Figure 3 shows the luminosity-redshift plane for the BAT blazars in comparison with the Seyfert-like AGNs detected by BAT. There are a few things that can be noted. First, the whole BAT sample of AGNs (Seyferts and blazars) spans almost four decades in redshift and eight in luminosity. There are 16 objects detected at redshift larger than one and they are all FSRQs. On the other hand, no blazars are detected at low luminosities and low redshift. This will be discussed in the following sections.

We cross-correlated the BAT blazar sample with the Third EGRET catalog (Hartman et al. 1999) using a two degree search radius around the BAT positions to cope with the large error radius of the EGRET positions. We further made sure, by searching in the literature, that the EGRET source, if any, is securely associated with the BAT counterpart (e.g., SowardsEmmerd et al. 2004). We found that only 12 BAT sources are included in the EGRET catalog being these three BL Lac and nine FSRQ objects (see Table 2). Moreover, we also checked how many BAT blazars were also detected by Fermi-Large Area Telescope (LAT) in the first three months of operations (Abdo 2009b). Again we found that only 12 sources (nine FSRQs and three BL Lac objects) are in common between the two samples (see Table 2). This implies that EGRET/Fermi and BAT do not sample exactly the same blazar population. In particular, the blazars BAT detects at the highest redshifts' are not (yet) present in the EGRET/Fermi catalogs. This 

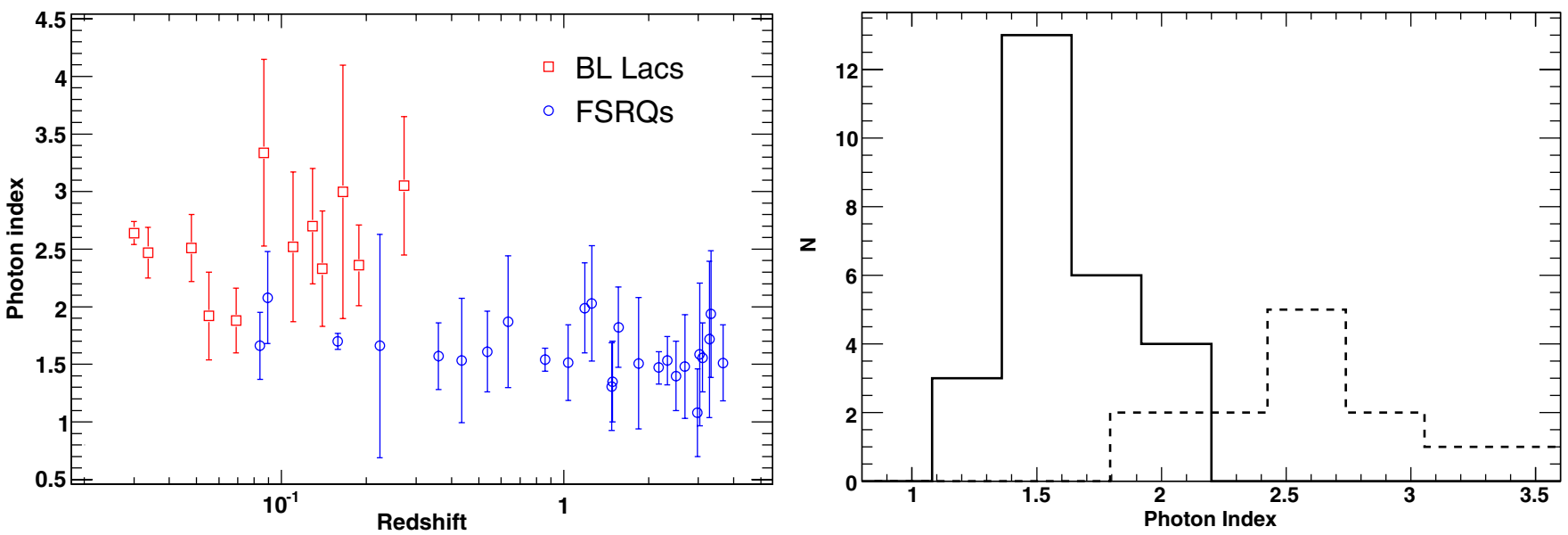

Figure 2. Left panel: photon index vs. redshift for the blazars in the BAT sample. The low-redshift sources, mainly BL Lac objects (red squares), have a soft spectrum (photon index of $\sim 2.5$, while the high-redshift ones (mainly FSRQs, blue circles) have a hard spectrum. Right panel: photon index distribution for FSRQs (solid line) and BL Lac objects (dashed line).

(A color version of this figure is available in the online journal.)

would mean that the high-redshift BAT blazars are faint in the $\mathrm{GeV}$ band. This hypothesis will be discussed in the following sections.

We believe the incompleteness of our blazar catalog is negligible. The fraction of unidentified sources in the total extragalactic sample is $<5 \%$. The most simple assumption is that these sources are distributed in source classes exactly like the identified sources. However, this does not take into consideration other properties such as radio emission. Indeed, no blazars are known to be radio-quiet sources (Wolter \& Celotti 2001). On this basis we found that none of the unidentified sources has a radio-loud object within $8^{\prime}$ or is present in the blazar catalogs used above. In addition, we also looked for spatial coincidences, within $8^{\prime}$ between the BAT unidentified objects and the CRATES catalog (Healey et al. 2007). The CRATES catalog contains all known FSRQs object above a $4.8 \mathrm{GHz}$ flux of $65 \mathrm{mJy}$ and for $|b|>10$ degrees. Again we found that none of the BAT unidentified objects is associated with a CRATES radio source. We, thus, confidently believe that the incompleteness of the BAT blazar sample is negligible (i.e., no new blazars are hiding among the unidentified BAT sources).

\section{THE EVOLUTION OF BLAZARS}

We first test the evolution of the BAT blazars by applying the $V / V_{\text {MAX }}$ method proposed by Schmidt (1968). For a non-evolving source population, $V / V_{\mathrm{MAX}}$ is expected to be uniformly distributed between 0 and 1 . Thus, in the case of no evolution the average $V / V_{\operatorname{MAX}}$ is 0.5 . The error on the average value can be computed as $\sigma=1 /(12 N)^{1 / 2}$. A value of the $\left\langle V / V_{\mathrm{MAX}}\right\rangle$ significantly different from 0.5 indicates positive (if $>0.5$ ) or negative evolution (otherwise). Computing the $\left\langle V / V_{\operatorname{MAX}}\right\rangle$ for the whole sample of blazars we obtain $0.666 \pm 0.045$. This indicates that the blazars population detected by BAT evolves positively at $>3 \sigma$. This means that the luminosity or density of the blazars is increasing with redshift. Moreover, we computed the $\left\langle V / V_{\mathrm{MAX}}\right\rangle$ for FSRQs and BL Lac objects separately in order to test if the two subpopulations evolve differently. We find a value of $\left\langle V / V_{\mathrm{MAX}}\right\rangle$ of $0.728 \pm 0.056$ and $0.576 \pm 0.083$ for FSRQs and BL Lac objects, respectively. Thus, this preliminary analysis shows that while FSRQs are evolving strongly BL Lac objects show only

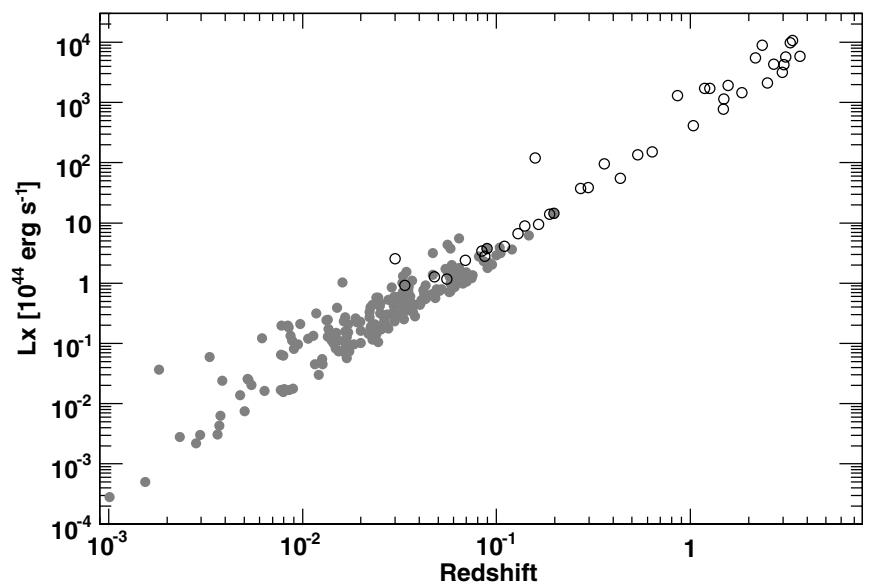

Figure 3. Luminosity-redshift plane for the BAT blazars (empty circles) and BAT Seyfert-like AGNs (filled gray circles).

mild evolution (compatible at $\sim 2 \sigma$ with no evolution). As a control sample, we used the sample of the Seyfert-like AGNs detected by BAT. Since this population is truly local (Ajello et al. 2008c; Tueller et al. 2008), we expect the $\left\langle V / V_{\mathrm{MAX}}\right\rangle$ to return a test value of 0.5 . As expected, we obtain $0.509 \pm 0.021$. These results are summarized in Table 3.

Another test of cosmological evolution, although less powerful than the $V / V_{\text {MAX }}$ method, is the $\log N-\log S$ test which is based on the distribution of source counts above a given flux. This distribution is generally expressed as $N(>S)=A S^{-\beta}$, where $S$ is the source flux. In this test, a non-evolving population shows a distribution which is consistent with an Euclidean distribution with $\beta=3 / 2$. If the source population is positively evolving then a $\beta>3 / 2$ is expected. The results of the $\log N-$ $\log S$ test are in excellent agreement with those of the $\left\langle V / V_{\mathrm{MAX}}\right\rangle$ method (see Table 3). They confirm that while the Seyfert population is not evolving, the blazar populations (and in particular the FSRQs) are evolving positively. Also the normalization of the $\log N-\log S$ distributions (e.g., A parameter in Table 3) highlights that, at the current flux limit of BAT, blazars are five times rarer than normal Seyfert galaxies. Thus, it is only because of the uniformly deep all-sky exposure that BAT gathered a sizable sample of them. 
Table 2

Blazar Sample

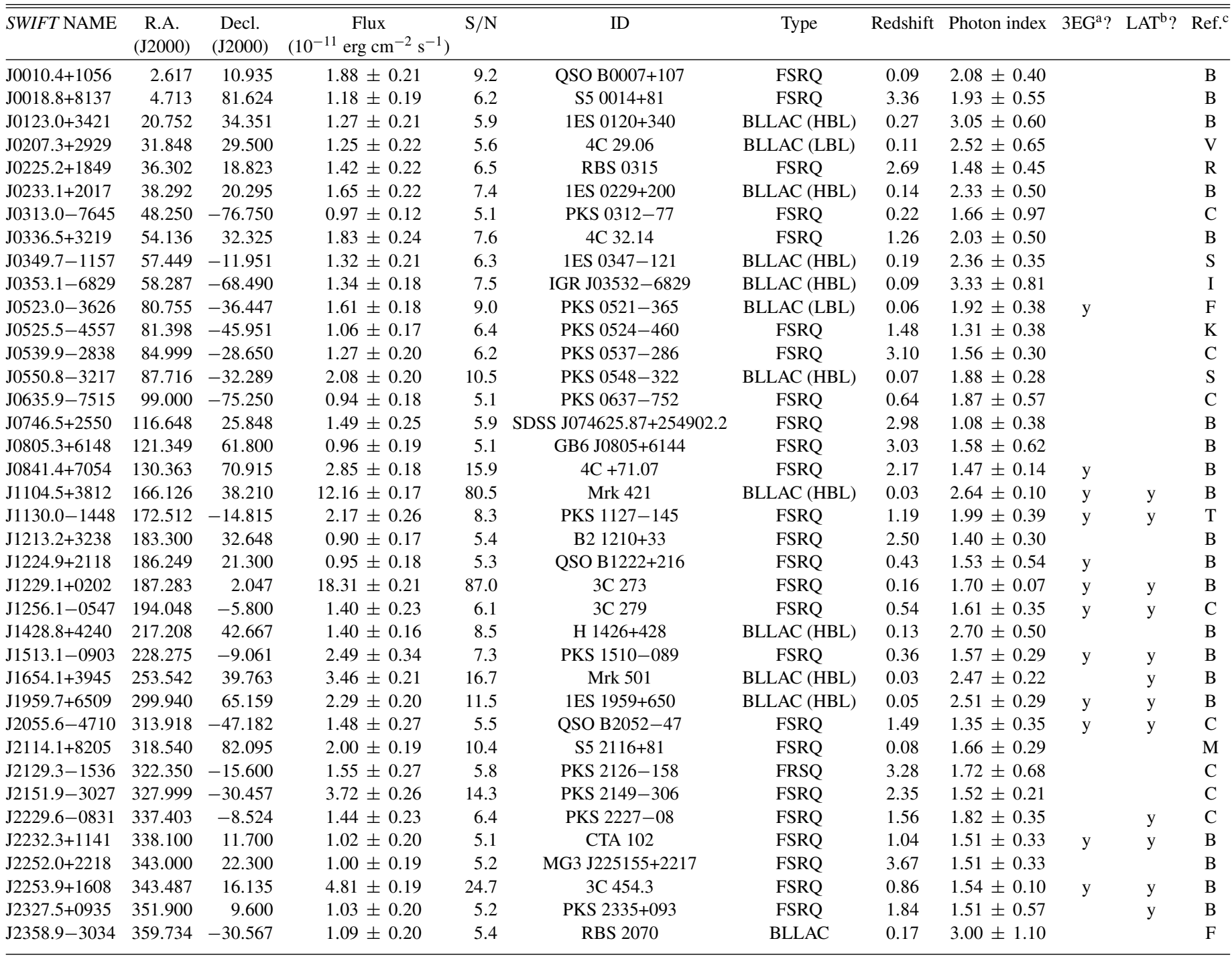

Notes.

${ }^{a}$ Counterpart in the third EGRET catalog (Hartman et al. 1999)?

${ }^{\mathrm{b}}$ Detected by Fermi-LAT (Abdo 2009b)?

${ }^{c}$ References for the optical classification and redshift: $\mathrm{B}=$ Massaro et al. (2009), $\mathrm{F}=$ Falomo et al. (1994), $\mathrm{C}=\mathrm{Healey}$ et al. (2008), I=Masetti et al. (2006), K=Stickel et al. (1993), $\mathrm{M}=$ Marcha et al. (1996), $\mathrm{R}=\mathrm{Schwope}$ et al. (2000), $\mathrm{S}=$ Sbarufatti et al. (2005), $\mathrm{T}=$ Stanghellini et al. (1998), V=Véron-Cetty \& Véron (2006).

Table 3

$V / V_{\text {MAX }}$ and $\log N-\log S$ Tests

\begin{tabular}{lcccr}
\hline \hline \multicolumn{1}{c}{ Sample } & $\left\langle V / V_{\text {MAX }}\right\rangle$ & $\beta^{\mathrm{a}}$ & $A^{\mathrm{b}}$ & No. of Objects \\
\hline Seyferts & $0.509 \pm 0.021$ & $1.496 \pm 0.073$ & $6.70 \pm 0.48$ & 199 \\
BLAZARs & $0.666 \pm 0.045$ & $1.932 \pm 0.206$ & $1.27 \pm 0.20$ & 38 \\
FSRQs & $0.728 \pm 0.056$ & $2.077 \pm 0.269$ & $0.83 \pm 0.16$ & 26 \\
BL Lac objects & $0.576 \pm 0.083$ & $1.694 \pm 0.316$ & $0.38 \pm 0.10$ & 12 \\
\hline
\end{tabular}

Notes.

${ }^{\text {a }}$ Best-fit exponent of the $\log N-\log S$ distribution (e.g., $N(>S)=A S^{-\beta}$ ).

${ }^{\mathrm{b}}$ Surface density of objects above $10^{-11} \mathrm{erg} \mathrm{cm}^{-2} \mathrm{~s}^{-1}$ in units of $10^{-3} \mathrm{deg}^{-2}$.

\subsection{The Evolving Luminosity Function}

The differential luminosity function of a population of objects is defined as the number of objects per unit comoving volume and per unit luminosity interval:

$\Phi\left(L_{X}, z\right)=\frac{d^{2} N}{d V d L_{X}}\left(L_{X}, V\right) \times \frac{d V}{d z}=\rho\left(L_{X}, V\right) \times \frac{d V}{d z}$, where $d V / d z$ is the comoving volume element per unit redshift and unit solid angle (see, e.g., Hogg 1999). The present day XLF can be obtained for $z=0$ as $\Phi\left(L_{X}, z=0\right)$. We model the present day XLF with two commonly used functions. A simple power of the form

$$
\Phi\left(L_{X}, z=0\right)=\frac{d N}{d L_{X}}=\frac{A}{L_{*}}\left(\frac{L_{X}}{L_{*}}\right)^{-\gamma_{2}},
$$


and a double power law of the form (see, e.g., Ueda et al. 2003; Hasinger et al. 2005)

$\Phi\left(L_{X}, z=0\right)=\frac{d N}{d L_{X}}=\frac{A}{\ln (10) L_{X}}\left[\left(\frac{L_{X}}{L_{*}}\right)^{\gamma_{1}}+\left(\frac{L_{X}}{L_{*}}\right)^{\gamma_{2}}\right]^{-1}$,

where in Equation (3) we set $L_{*}=10^{44} \mathrm{erg} \mathrm{s}^{-1}$, while in Equation (4) $L_{*}$ is allowed to vary.

\subsubsection{The Luminosity and Density Evolutions}

The simplest scenarios of evolution are pure luminosity evolution (PLE) and pure density evolution (PDE). In the PLE case, the XLF becomes

$$
\Phi\left(L_{X}(z), z\right)=\Phi\left(L_{X} / e(z), z=0\right),
$$

while in the PDE case

$$
\Phi\left(L_{X}, z\right)=\Phi\left(L_{X}, z=0\right) \times e(z)
$$

and the evolution is parameterized using the common power-law evolutionary factor:

$$
e(z)=(1+z)^{k+\gamma z} .
$$

This is thus clear that in the PLE case the typical blazar luminosity is changing with redshift, while in the PDE case only their densities are changing with redshift. We note that this formulation of the evolutionary factor (suggested first by Wall et al. 2008) reduces for $\gamma=0$ to the standard power law $(1+z)^{k}$. For clarity, we call PDE (PLE) or modified-PDE/PLE (or MPDE, MPLE) the case in which $\gamma$ has been fixed to zero or was allowed to vary.

\subsection{Maximum Likelihood Analysis}

A classical approach to derive the XLF is based on the $1 / V_{\text {MAX }}$ method of Schmidt (1968). However, this method is known to introduce a bias if there is significant evolution within bins of redshift. Moreover, considering the small number of objects in our sample, binning would result in a loss of information. We thus decided to apply the maximum likelihood method using the formalism introduced by Marshall et al. (1983).

In this method, the luminosity-redshift plane is parsed into extremely small intervals of size $d L_{X} d z$. In each element, we compute the expected number of blazars with luminosity $L_{X}$ and redshift $z$ :

$$
\lambda\left(L_{X}, z\right) d L_{X} d z=\rho\left(L_{X}, V\right) \Omega\left(L_{X}, z\right) \frac{d V}{d z} d L_{X} d z,
$$

where $\Omega\left(L_{X}, z\right)$ is the sky coverage of the survey (see Section 2 for details). The sampling of the luminosity-redshift plane is sufficiently fine that in each $d L_{X} d z$ element the number of observed blazars is either 1 or 0 . In this sparse sampling limit, we can define a likelihood function based on joint Poisson probabilities where the Poisson model is

$$
f(x: m)=\frac{e^{-\mu} \mu^{x}}{x !},
$$

where $\mu$ is the expected number of blazars. If $x=1$ then the function is $\mu e^{-\mu}$, and if $x=0$ it is $e^{-\mu}$. In this case, the likelihood function can be written as

$$
L=\prod_{i} \lambda\left(L_{X, i}, z_{i}\right) d L_{X} d z e^{-\lambda\left(L_{X, i}, z_{i}\right) d L_{X} d z} \times \prod_{j} e^{-\lambda\left(L_{X, j}, z_{j}\right) d L_{X} d z} .
$$

This is the combined probability of observing one blazar at each element $\left(L_{X, i}, z_{i}\right)$ populated by one BAT blazar and observing zero blazars everywhere else $\left(L_{X, j}, z_{j}\right)$. Transforming to the standard expression $S=-2 \ln L$ and dropping terms which are not model dependent, we obtain

$$
\begin{aligned}
S= & -2 \sum_{i} w_{i} \ln \left[\rho\left(L_{X, i}, z_{i}\right)\right] \\
& +2 \int_{L_{X, \min }}^{L_{X, \max }} \int_{z_{\min }}^{z_{\max }} \lambda\left(L_{X, i}, z_{i}\right) d L_{X} d z,
\end{aligned}
$$

where following Borgani et al. (2001) we have introduce a weighting term $\omega_{i}$ which takes into account the uncertainties in the luminosity of each single blazar. In this way, each blazar instead of being a point in the $L_{X}, z$-plane is smoothed in the $L_{X}$ direction according to a Gaussian distribution with a width set by the $1 \sigma$ luminosity error. Thus, a weight is assigned to each element in the luminosity-redshift plane based on the fractional contributions of all blazars in the same redshift interval:

$$
w_{i}=\sum_{k} \frac{1}{\sqrt{2 \pi \epsilon_{L_{X, k}}^{2}}} \exp \left[-\frac{\left(L_{X, k}-L_{X, i}\right)^{2}}{2 \epsilon_{L X, k}^{2}}\right] d L_{X},
$$

where the summation $k$ is over the blazars with a redshift between $z_{i}-d z / 2$ and $z_{i}+d z / 2$. The limits of integration of Equation (11), unless otherwise stated, are $L_{X \text {, } \min }=10^{41} \mathrm{erg}$ $\mathrm{s}^{-1}, L_{X, \max }=10^{50} \mathrm{erg} \mathrm{s}^{-1}, z_{\min }=0$, and $z_{\max }=6$. While, the results are independent of the upper limits of integration, as far as they are chosen to be large, this is not the case for the value of $L_{X \text {,min }}$ if the local XLF is modeled as a single power law. In this case, the lower limit of integration needs to be set to the minimum observed blazar luminosity $\left(6 \times 10^{43} \mathrm{erg} \mathrm{s}^{-1}\right.$ and $2 \times 10^{44} \mathrm{erg} \mathrm{s}^{-1}$ for BL Lac objects and FSRQs, respectively).

The best-fit parameters are determined by minimizing ${ }^{9} S$ and their associated $1 \sigma$ errors are computed by varying the parameter of interest, while the others are allowed to float, until an increment of $\Delta S=1$ is achieved. This gives an estimate of the $68 \%$ confidence region for the parameter of interest (Avni 1976).

\subsection{Alternative Maximum Likelihood Formulation}

Another way of posing the maximum likelihood problem is (following e.g., Chiang \& Mukherjee 1998; Narumoto \& Totani 2006)

$$
L=\exp \left(-N_{\mathrm{exp}}\right) \prod_{i=1}^{N_{\mathrm{obs}}} \Phi\left(L_{X, i}, z_{i}\right)
$$

where $N_{\exp }$ is the expected number of blazar detections:

$$
N_{\exp }=\int d z \int d L_{X} \Phi\left(L_{X}, z\right) .
$$

In this case, the function $S(=-2 \ln L)$ is defined as

$$
S=-2 \sum_{i}^{N_{\text {obs }}} \ln \left(\Phi\left(L_{X, i}, z_{i}\right)\right)-2 N \ln \left(N_{\text {exp }}\right) .
$$

We tested that we get exactly the same results if we use one or the other formulation of the maximum likelihood problem. Thus, the results that we present in the following sections are independent of the maximum likelihood (ML) function chosen.

\footnotetext{
9 The MINUIT minimization package, embedded in ROOT (root.cern.ch), has been used for this purpose.
} 
Table 4

Parameters of Fitted Luminosity Functions

\begin{tabular}{|c|c|c|c|c|c|c|c|c|c|c|c|c|}
\hline Sample & No. of Objects & Model No. & Model & $A^{\mathrm{a}}$ & $\gamma 1$ & $\gamma^{2}$ & $L_{*}$ & $k$ & $\gamma$ & $\mathrm{KS}_{z}$ & $\mathrm{KS}_{L_{X}}$ & $\mathrm{CXB} \%$ \\
\hline Seyferts & 199 & 1 & PLE+2pow & $0.909 \pm 0.064^{b}$ & $0.80 \pm 0.08$ & $2.67 \pm 0.20$ & $0.61 \pm 0.18$ & 0 & $\ldots$ & 0.99 & 0.94 & $21.6 \%$ \\
\hline Seyferts & 199 & 2 & PLE+2pow & $0.778 \pm 0.055^{\mathrm{b}}$ & $0.84 \pm 0.08$ & $3.01 \pm 0.30$ & $0.61 \pm 0.14$ & $2.62 \pm 1.18$ & $\ldots$ & 0.99 & 0.93 & $55.2 \%$ \\
\hline BLAZAR $^{\mathrm{c}}$ & 38 & 3 & PDE+1pow & $0.757 \pm 0.135$ & $\ldots$ & $2.67 \pm 0.13$ & 1.0 & $4.00 \pm 0.77$ & $\ldots$ & 0.43 & 0.22 & $22.0 \%$ \\
\hline BLAZAR $^{c}$ & 38 & 4 & MPDE+1pow & $0.732 \pm 0.117$ & $\cdots$ & $3.08 \pm 0.20$ & 1.0 & $8.95 \pm 1.90$ & $-0.69 \pm 0.27$ & 0.34 & 0.40 & $318.0 \%$ \\
\hline BLAZAR $^{c}$ & 38 & 5 & MPLE+1pow & $0.804 \pm 0.131$ & $\ldots$ & $3.13 \pm 0.21$ & 1.0 & $2.96 \pm 0.47$ & $-0.23 \pm 0.08$ & 0.43 & 0.41 & $18.4 \%$ \\
\hline BLAZAR & 38 & 6 & PDE+1pow & $0.255 \pm 0.041$ & $\ldots$ & $2.26 \pm 0.07$ & 1.0 & $2.05 \pm 0.57$ & $\ldots$ & 0.002 & 0.00 & $6.9 \%$ \\
\hline BLAZAR & 38 & 7 & MPLE+2pow & $1.379 \pm 0.224$ & $-0.87 \pm 1.31$ & $2.73 \pm 0.38$ & $1.81 \pm 0.77$ & $3.45 \pm 0.44$ & $-0.25 \pm 0.07$ & 0.86 & 0.88 & $\sim 20.0 \%$ \\
\hline BLAZAR & 38 & 8 & MPDE+2pow & $0.948 \pm 0.152$ & $-0.83 \pm 1.43$ & $2.54 \pm 0.21$ & $1.95 \pm 0.93$ & $11.62 \pm 1.40$ & $-0.85 \pm 0.18$ & 0.46 & 0.85 & $640.0 \%$ \\
\hline FSRQ $^{\mathrm{d}}$ & 26 & 9 & MPLE+1pow & $0.533 \pm 0.104$ & $\ldots$ & $3.45 \pm 0.20$ & 1.0 & $3.72 \pm 0.50$ & $-0.32 \pm 0.08$ & 0.86 & 0.87 & $9.0 \%$ \\
\hline FSRQ $^{\mathrm{e}}$ & 26 & 10 & MPLE+2pow & $0.175 \pm 0.034$ & $<-50.0$ & $2.49 \pm 0.37$ & $2.42 \pm 0.19$ & $3.67 \pm 0.48$ & $-0.30 \pm 0.08$ & 0.85 & 0.89 & $8.3 \%$ \\
\hline $\mathrm{BL} \mathrm{Lac}^{\mathrm{c}}$ & 12 & 11 & PLE+1pow & $0.830 \pm 0.240$ & $\ldots$ & $2.61 \pm 0.37$ & 1.0 & $-0.79 \pm 2.43$ & $\ldots$ & 0.55 & 0.33 & $0.3 \%$ \\
\hline $\mathrm{BL} \mathrm{Lac}^{\mathrm{c}}$ & 12 & 12 & PLE+1pow & $0.784 \pm 0.226$ & $\ldots$ & $2.73 \pm 0.17$ & 1.0 & 0 & $\ldots$ & 0.55 & 0.33 & $0.3 \%$ \\
\hline BL Lac & 12 & 13 & PLE+2pow & $1.506 \pm 0.435$ & $-0.89 \pm 3.7$ & $2.51 \pm 1.61$ & $1.78 \pm 2.71$ & $1.54 \pm 2.73$ & $\ldots$ & 0.71 & 0.93 & $2.2 \%$ \\
\hline
\end{tabular}

Notes. Parameters without an error estimate were kept fixed during the fitting stage. KS values are the probabilities of the model and the data to be drawn from the same parent population

a In unit of $10^{-7} \mathrm{Mpc}^{-3} \mathrm{erg}^{-1} \mathrm{~s}$ unless otherwise stated.

b In unit of $10^{-5} \mathrm{Mpc}^{-3}$

c $L_{\mathrm{Min}}$ has been set to $6 \times 10^{43} \mathrm{erg}^{-1} \mathrm{~s}$

d $L_{\text {Min }}$ has been set to $2 \times 10^{44} \mathrm{erg}^{-1} \mathrm{~s}$.

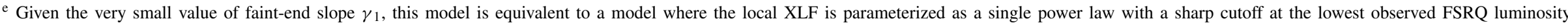
of $\sim 2 \times 10^{44} \mathrm{erg} \mathrm{s}^{-1}$. 


\subsection{Consistency Checks}

The maximum likelihood approach does not provide a goodness-of-fit test and this implies that other methods have to be used to understand if the fitted function is a good representation of the data. A common procedure is to use the Kolmogorov-Smirnov (KS) test which is based on the maximum distance $\left(D_{\mathrm{KS}}\right)$ between the (cumulative) distributions under comparison. This test computes the probability of observing the KS test statistics as large or larger than the observed one and it can be used to reject a model when too low. We apply a KS test to both the cumulative redshift and luminosity distributions of the BAT blazars and we reject XLF models which produce a KS probability $<20 \%$.

As a further test we check that the best-fit XLF reproduces well the observed source count distribution (also known as $\log N-\log S$ ). The all-sky number of blazars with a flux stronger than $S$ can be computed as

$$
N(>S)=4 \pi \int_{0}^{z_{\max }} d z \frac{d V}{d z} \int_{L_{X(z, S)}}^{\infty} d L_{X} \rho\left(L_{X}, V(z)\right),
$$

where $L_{X(z, S)}$ is the luminosity of a blazar at redshift $z$ whose flux is $S$.

\subsection{The Cosmic X-ray Background Constraint}

This is almost certain that the bulk of the CXB emission (below $200 \mathrm{keV}$ ), even if presently unresolved above $10 \mathrm{keV}$, is due to Seyfert-like AGNs (e.g., Ueda et al. 2003; La Franca et al. 2005; Treister \& Urry 2005; Gilli et al. 2007; Silverman et al. 2008). Even though one of our goal is to estimate, in the most robust way, the contribution of blazars to the CXB spectrum, as a first step the CXB emission can be used to reject invalid XLF models. Indeed the blazar contribution to the CXB emission at $\mathrm{X}$-ray energies is expected to be $\sim 10 \%$ (e.g., Giommi et al. 2006). A much larger fraction would conflict with the present estimates produced by population synthesis models (e.g., Ueda et al. 2003; Treister \& Urry 2005; Gilli et al. 2007) and can be used to rule out a given evolutionary model. Thus, for each best-fit XLF model, we compute the integrated background flux arising from the blazar population. This flux can be derived as

$$
F_{\mathrm{CXB}}=\int_{z_{\min }}^{z_{\max }} d z \frac{d V}{d z} \int_{L_{X, \min }}^{L_{X, \max }} d L_{X} F_{X}\left(L_{X}, z\right) \rho\left(L_{x}, V(z)\right),
$$

where the limits of integration are the same as in Equation (11) and $F_{X}\left(L_{X}, z\right)$ is the flux of a source with luminosity $L$ at redshift $z$.

As a final note, we remark that we are not interpreting the CXB as a "hard constraint" in the sense that the integrated blazar emission of Equation (17) is not constrained to be $\sim 10 \%$, but a model XLF which overproduces the entire CXB (e.g., producing more than $100 \%$ of the CXB), in the $15-55 \mathrm{keV}$ band, can be certainly ruled out.

\section{RESULTS}

\subsection{The Control Sample: Local Seyfert Galaxies}

Recently, Tueller et al. (2008) computed the (non-evolving) XLF of local Seyfert galaxies using a sample of 88 objects detected by BAT in the first nine months of operations. Given the small redshift range spanned $(z \leqslant 0.1)$, they did not test for the evolution of Seyferts in the local universe. However, it is well established that the population of radio-quiet AGNs evolves in density and luminosity (e.g., Hasinger et al. 2005; Silverman et al. 2008). Thus, the sample of 199 Seyferts detected in this analysis represents a good test for the ML method introduced in Section 3.2. Here, we aim at deriving a parametric representation of the Seyfert XLF testing at the same time for their evolution in the local universe.

We model the local XLF (e.g., $\Phi\left(L_{X}, z=0\right)$ ) with a double power-law model as in Equation (4) and fix the evolutionary term $k$, of the PLE, to zero. The best-fit parameters, reported in Table 4 (model 1), are $\gamma_{1}=0.80 \pm 0.08, \gamma_{2}=2.67 \pm 0.20$, and $L^{*}=6.1 \pm 1.4 \times 10^{43} \mathrm{erg} \mathrm{s}^{-1}$ and are in very good agreement with the values reported by Tueller et al. (2008). ${ }^{10}$ The error bars are generally smaller because the sample we use is larger and because the fit is done to the unbinned data set. As shown by the redshift, luminosity, and $\log N-\log S$ distributions (reported in Figures 4 and 5), this non-evolving XLF model is an highly acceptable description of the data set (KS tests $\sim 1$; see Table 4 ).

Allowing the XLF to evolve in luminosity (see model 2 in Table 4) produces an equally good fit with an evolution parameter $(k=2.62 \pm 1.18)$ which denotes positive evolution although constrained only at the $\sim 2 \sigma$ level. It is interesting to note that the evolution parameter is in good agreement with the values of $2.29 \pm 0.09$ and $2.7 \pm 0.2$ found for the PLE case by Ueda et al. (2003) and Hasinger et al. (2005), respectively. However, since in our case the two models produce an equally good fit (see KS test values), the non-evolving XLF has to be preferred because of the lower number of free parameters. Thus, we believe that the evidences of the evolution of radio-quiet AGN in the local universe are, with the current data set, marginal. As a final proof, we built a nonparametric representation of the luminosity function of the Seyferts using the $1 / V_{\text {MAX }}$ method (Schmidt 1968). In order to test for evolution, we binned the data set in two redshift bins containing approximately the same number of sources. This luminosity function is reported in Figure 6. An evolving XLF would show a shift (in luminosity or density) from one redshift bin to the other. This is not the case for the BAT Seyferts whose luminosity functions, derived in two different redshift bins, are the continuation one of each other. In the same figure, the best-fit non-evolving XLF (model 1) is also displayed and it is clear that this represents the data well. The fact that the evolution, if detected, is only marginal is not surprising, but consistent with the results of the $V / V_{\text {MAX }}$ test reported in Table and the Euclidean behavior of the $\log N-\log S$ as reported by Ajello et al. (2008c) and Tueller et al. (2008).

\subsection{A Single Blazar Population}

As a first case, we start assuming that the local (present day) XLF can be adequately approximated by a simple power law as in Equation (3). It was already shown by Marshall et al. (1983) that in this case it is impossible to discriminate between density and luminosity evolution. So we refer to density evolution, but we note that the two type of evolutions are formally equivalent in this case.

In the simple power-law case (and PDE), we obtain a slope of the XLF of $\gamma_{2}=2.67 \pm 0.13$ and an evolution parameter of $4.00 \pm 0.77$. The error on the evolution parameter $k$ confirms that the evolution is significantly detected in the BAT sample (see model 3 in Table 4). We note that the XLF slope is in

\footnotetext{
10 The different value of $H_{0}$ and energy band that Tueller et al. (2008) adopt produce the net effect that the luminosities quoted here are directly comparable to those quoted by Tueller et al. (2008) without the need of a conversion factor (i.e., the conversion factor is $\sim 1$ )
} 

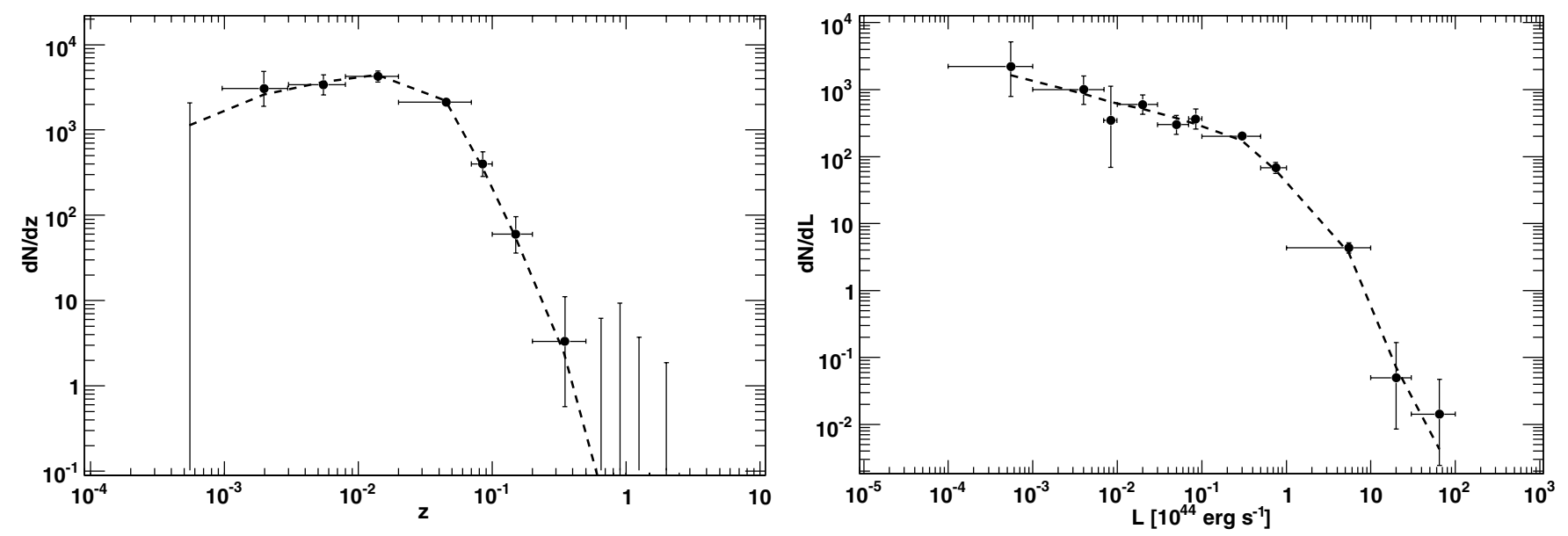

Figure 4. Redshift (left) and luminosity (right) distribution of the BAT Seyferts. Long error bars consistent with zero are $1 \sigma$ upper limits in the case of observing zero events (Gehrels 1985). For both cases, the dashed line represents a non-evolving XLF (model 1 in Table 4) convolved with the BAT sky coverage.

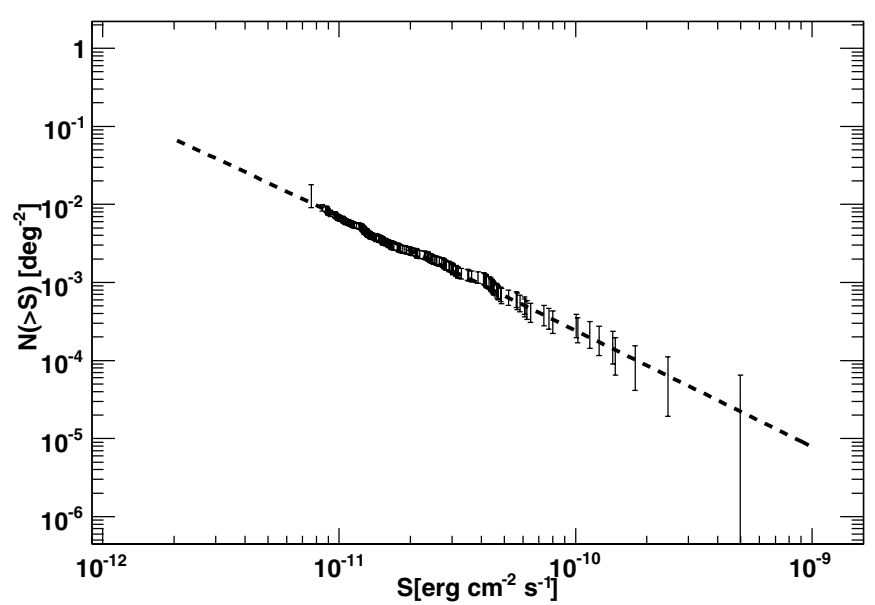

Figure 5. Cumulative $\log N-\log S$ of the BAT Seyferts. The dashed line is the prediction of the best-fit XLF (model 1 in Table 4).

very good agreement with the bright-end slope of the Seyfertlike AGNs detected by BAT (see Table 4). Figure 7 shows the redshift and luminosity distribution of the BAT blazars with superimposed the best-fit XLF. The KS test shows that this is already an acceptable description of the data.

We note, from the left panel of Figure 7, that the best-fit PDE XLF fails to describe the drop in blazar counts above $z \sim 4$. This might be a sign of a possible cutoff in the evolution. Thus, we decided to model the evolution factor as $e(z)=(1+z)^{k+\gamma z}$ as done by Wall et al. (2008; see Equation (7)) calling this model a modified PDE (or MPDE). The best fit to the data shows that the value of $\gamma$ is constrained to be negative at the $\sim 2.5 \sigma$ level $(\gamma=-0.69 \pm 0.27)$. This shows that a cutoff in the evolution is needed in our data. Although, this model reproduces our data reasonably well, the integration of the XLF shows that it overpredicts the "total" CXB emission by a factor 3 (see Table 4). Thus, the interpretation of density evolution might not be the correct one. A similar effect was already noted by Marshall et al. (1983) who concluded for their sample of optical quasi-stellar objects (QSOs) that luminosity evolution was likely occurring.

As already said, for a single power-law XLF there is no formal difference between density or luminosity evolution. The only difference is that in the integral of Equation (11), the integration limit $L_{X, \min }$ is evolving and can be expressed

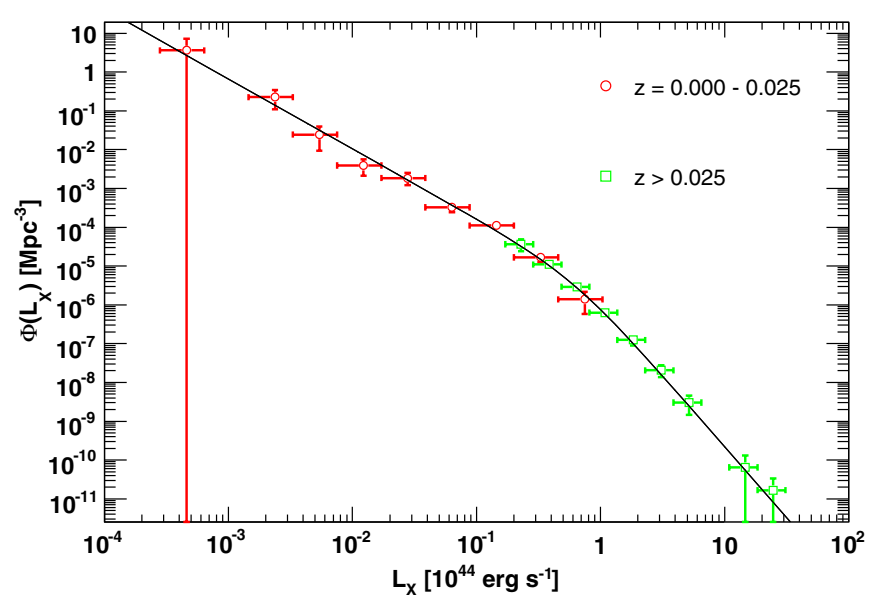

Figure 6. Luminosity function of the Seyfert-like AGNs, derived using the $1 / V_{\text {MAX }}$ method, in two redshift bins (data points). The solid line is a nonevolving XLF double power-law model (model 1 in Table 4) as derived from the ML algorithm.

(A color version of this figure is available in the online journal.)

as $L_{X, \min }=L_{X, \min }^{0} \times e(z)$, where $L_{X, \text { min }}^{0}$ is the present day luminosity cutoff $\left(\right.$ e.g., $\left.6 \times 10^{43} \mathrm{erg} \mathrm{s}^{-1}\right)$ and $e(z)=(1+z)^{k+\gamma z}$. The best fit confirms that indeed the luminosity evolution is a better interpretation of the underlying evolution. Indeed, integrating the XLF we get that the blazar population, described by this MPLE function, accounts for $20 \%$ of the CXB emission in the $15-55 \mathrm{keV}$ band. We also note that the best-fit value of the evolution parameter $k=2.96 \pm 0.46$ is in very good agreement with what found by Ueda et al. (2003) for X-ray-selected AGNs and by Wolter \& Celotti (2001) for X-ray-selected FSRQs.

All the XLF models described so far (models 3, 4, and 5 in Table 4) become unacceptable if the limit on the minimum observed luminosity $L_{\min }$ (see Equation (11)) is removed. Indeed, in this case (see results of the KS tests for model 6), the luminosity and redshift distributions are not reproduced correctly because the best-fit model predicts many blazars at low luminosity and low redshift which are not detected by BAT. Thus, a rather drastic change in the power-law behavior of the local XLF is required in order to reproduce the lack of lowluminosity objects.

To test this scenario, we model the local XLF as a double power law (see Equation (4)) coupled to a MPLE model. In 

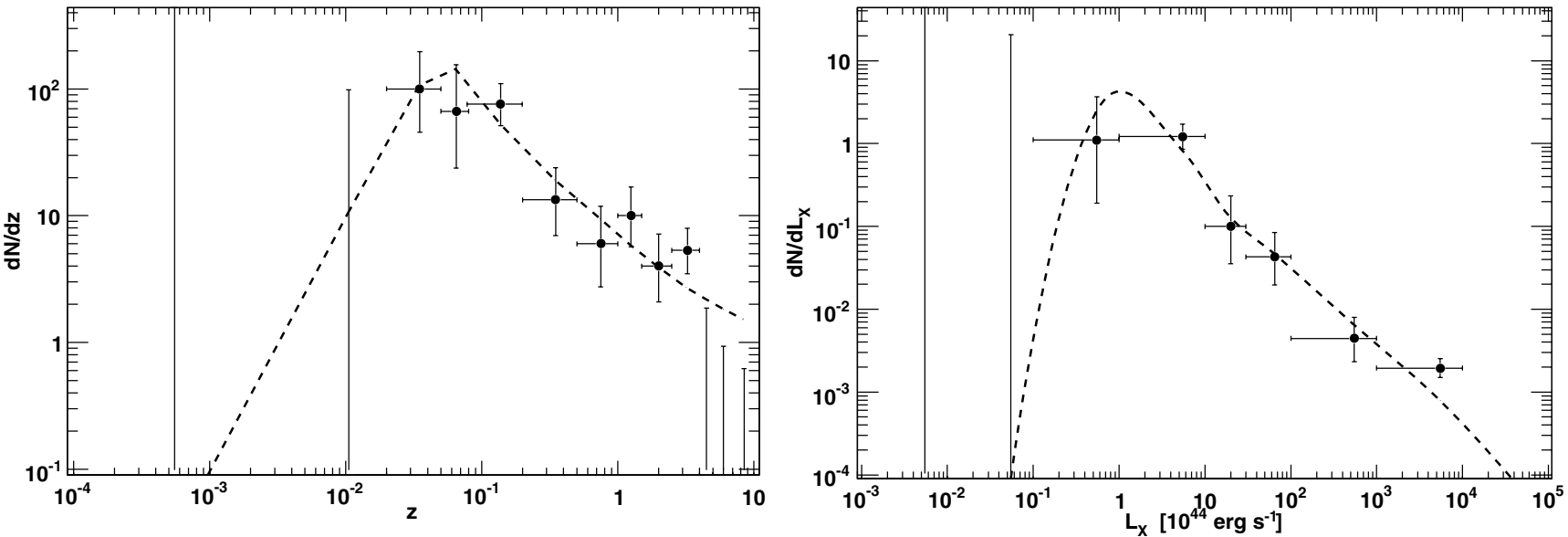

Figure 7. Redshift (left) and luminosity (right) distribution of the BAT blazars with superimposed (dashed line) the prediction of the best-fit PDE XLF (single power-law case, see model 3 in Table 4) convolved with the BAT sky coverage. Error bars were computed taking into account the Poisson error (Gehrels 1985). Long error bars consistent with zero are $1 \sigma$ upper limits for the case of observing zero events in a given bin.
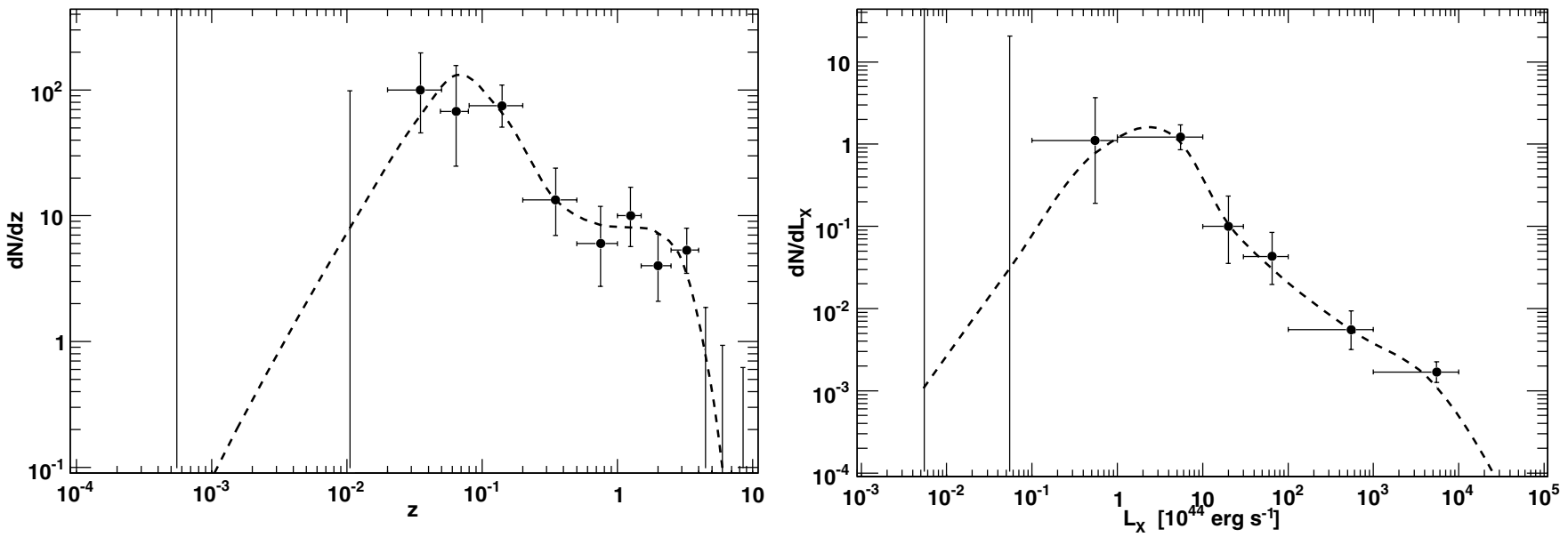

Figure 8. Redshift (left) and luminosity (right) distribution of the BAT blazars. Error bars were computed taking into account the Poisson error (Gehrels 1985). For both cases, the dashed line represents the MPLE XLF (double power-law model, see model 7 in Table 4) convolved with the BAT sky coverage.
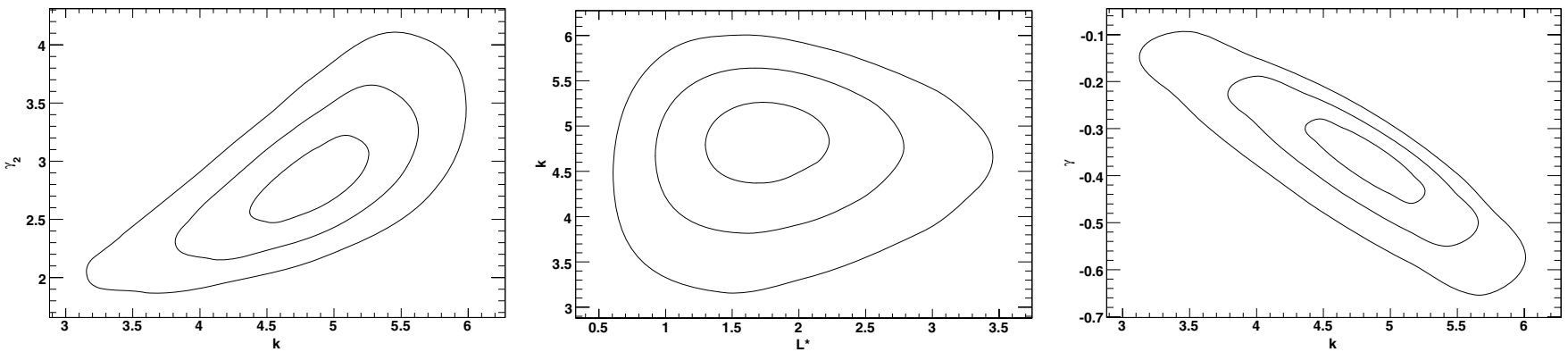

Figure 9. Confidence contours $(1 \sigma, 2 \sigma$, and $3 \sigma)$ for the bright-end XLF slope $\left(\gamma_{2}\right)$, the evolution parameters $(k$ and $\gamma)$ and the break luminosity $L_{*}$ for the best-fit XLF model (model 7 in Table 4).

this model, we remove the constraint of a low-luminosity cutoff and the fit is performed to the whole luminosity-redshift plane. This XLF model reproduces our data accurately (see model 7 in Table 4 and the distributions reported in Figure 8). Given the lack of low-luminosity objects, the faint-end slope $\gamma_{1}$ is, from our fit, required to be flat, but poorly constrained $(-0.87 \pm 1.31)$.

The slope of the bright-end part of the XLF $\gamma_{2}=2.73 \pm 0.37$ is in good agreement with the same slope found for the Seyferts (see model 1). The likelihood ratio test can be used to assess whether a model produces a significant improvement over another one. The likelihood ratio test is the difference between the value of $S$ (see Equation (11)) produced by different models. This value $(\Delta S)$ is expected to be asymptotically distributed as the $\chi_{n}^{2}$ (Wilks 1938) where $n$ is the difference between the degrees of freedom of the two models. The $\Delta S$ for model 7 (double power law plus MPLE) with respect to model 4 (single power law plus MPDE) is $\sim 10.1$ which translates in a probability of $0.0015^{11}$ that the improvement was obtained

11 The chance probability was computed using the $\chi^{2}$ distribution for 1 degree of freedom. Indeed, the difference between model 7 and model 4 is given by the faint-end slope $\gamma_{1}$ which is allowed to vary while the cutoff luminosity $L_{\min }$ (imposed in model 4) and the break luminosity $L_{*}$ (in model 7) represent essentially the same parameter. 

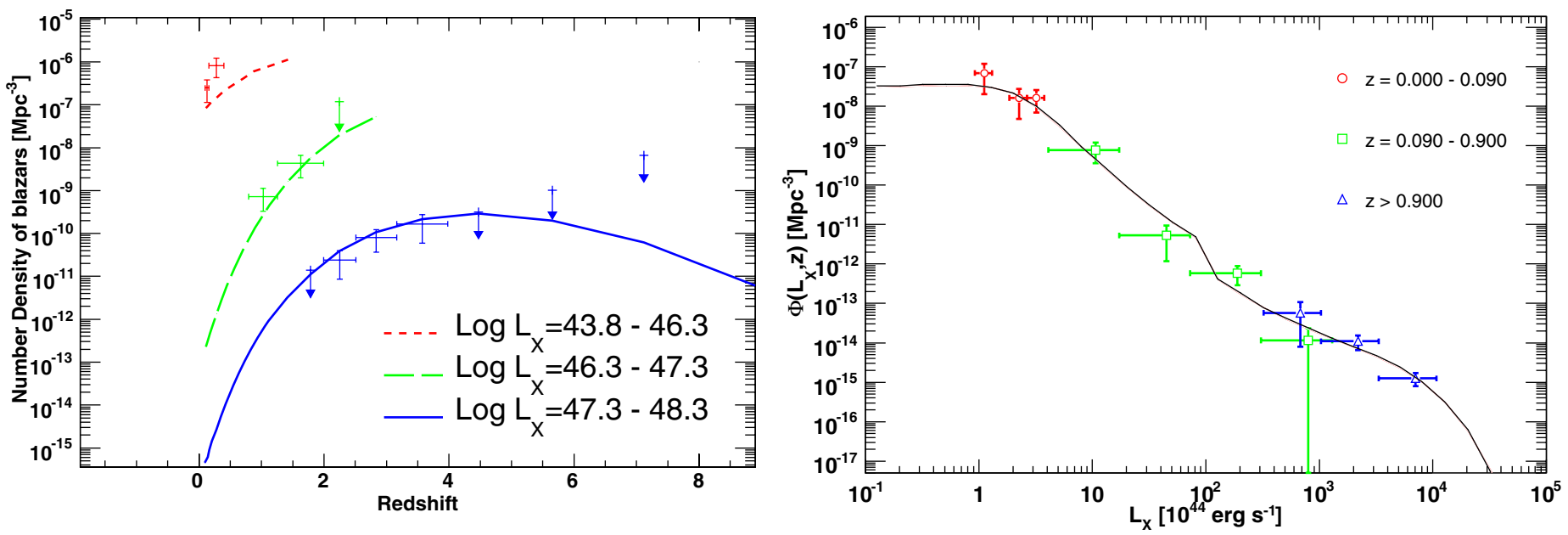

Figure 10. Left panel: number density of blazars (FSRQs and BL Lac objects) as a function of redshift and luminosity class. The solid lines represent the best-fit XLF model (model 7 in Table 4). The BAT data (points with errors) were "deconvolved" taking into account the BAT sensitivity (see Section 4.2 for details). Right panel: luminosity function of the BAT blazars built using the $1 / V_{\text {MAX }}$ method (datapoints) with superimposed the best-fit XLF model (model 7 in Table 4).

(A color version of this figure is available in the online journal.)

by chance. We also note that density evolution (with a double power law as a local XLF) reproduces the data equivalently well (see model 8), but it is ruled out since it overpredicts the CXB emission by a factor of $>5$. Thus, we consider model 7 as the best representation of our data. Figure 9 shows the confidence contours for the best-fit parameters.

The extreme flattening of the XLF at low luminosities can be the effect of beaming. As discussed by Urry \& Shafer (1984), relativistic beaming alters the observed luminosity function of blazars producing a flattening at low luminosities. For common jet emission scenarios (see Urry \& Shafer 1984, for details), the faint-end slope of the XLF should be $\sim 1.0$. Given the absolute lack of BAT blazars populating the low-luminosity part of the XLF, it is not surprising that the best-fit value of $\gamma_{1}$ is $\sim 1.5 \sigma$ away from the Urry \& Shafer (1984) prediction. On the other hand, relativistic beaming should not affect the bright-end slope which should reflect the slope of the intrinsic luminosity function. It thus becomes interesting to compare the value of $\gamma_{2}$ derived here with other surveys. Recently, Cara \& Lister (2008) derived the intrinsic radio luminosity function of the FanaroffRiley (FR) class II which is thought to be the parent population of FSRQs. They found that the slope of the intrinsic luminosity function is $2.53 \pm 0.06$ which is in good agreement with the value of $2.73 \pm 0.38$ derived here.

A visual representation of the best-fit XLF model (double power law plus MPLE model) is shown in the left panel of Figure 10 which reports the volume density of blazar as a function luminosity class and redshift. The datapoints are the "deconvolved" BAT observed data, that is the number (or density) of blazars which an instrument with optimum sensitivity would see. In order to deconvolve the BAT data, we computed for each bin of redshift and luminosity, the ratio between the integrals of $\Phi\left(L_{X}, z\right)$ and $\lambda\left(L_{X}, z\right)$ (see Equations (2) and (8) for a definition of both). This gives a correction factor which allows us to deconvolve the BAT data. Also note, that given the sparseness of the BAT data, the correction factor is sometimes averaged over large bins of redshift and luminosity where the XLF is strongly varying, thus it might be somewhat uncertain. Nevertheless, Figure 10 highlights that BAT is sampling with good accuracy the redshift peak of some of the most luminous objects in the universe. From the same figure, it is clear that the density of very luminous blazars $\left(\log L_{X}>10^{47} \mathrm{erg} \mathrm{s}^{-1}\right)$ peaks at large redshift and precisely at $z=4.3 \pm 0.5$. This is much larger than the value of $\sim 1.9$ derived (or assumed) for X-ray and Optical surveys (see, e.g., Ueda et al. 2003; Hasinger et al. 2005; Bongiorno et al. 2007; Silverman et al. 2008). The likely reason of this difference will be addressed in details in Section 6. The right panel of Figure 10 shows the nonparametric blazar XLF built using the $1 / V_{\text {MAX }}$ method along with the best-fit analytical XLF model (model 7). It is apparent the good agreement between the two representations.

\subsection{Two Populations: FSRQs and BL Lac Objects}

Previous works (e.g., Wolter et al. 1991; Rector et al. 2000; Wolter \& Celotti 2001; Beckmann et al. 2003; Padovani et al. 2007) have reported evidence about the different evolutionary behaviors of FSRQs and BL Lac objects. The $V / V_{\mathrm{MAX}}$ test reported in Section 3 showed that also in our sample the two classes of objects might evolve differently. In the following sections, we test this hypothesis.

\subsubsection{FSRQs}

We applied the two best-fit models of the previous section (MPLE coupled to a single and double power-law local XLF, respectively) to the FSRQ class. The best-fit parameters are reported in Table 4. We note that both XLF models produce essentially the same result. When the local XLF is modeled as a double power-law model, the faint-end slope $\gamma_{1}$ is required to be largely negative $(<-50)$ and the break luminosity $L_{*}$ coincides with the minimum observed luminosity of FSRQs in the BAT sample. Under this conditions, the double power-law model reduces to a single power-law distribution with a sharp cutoff at $L_{X}<2 \times 10^{44} \mathrm{erg} \mathrm{s}^{-1}$. Figures 11 and 12 (right panel) show how well the best-fit XLF models (models 9 and 10 in Table 4) reproduce the observed distributions (in redshift, luminosity, and source counts).

Figure 13 shows the number, and its volume density, of FSRQs in the universe for different luminosity classes as derived 

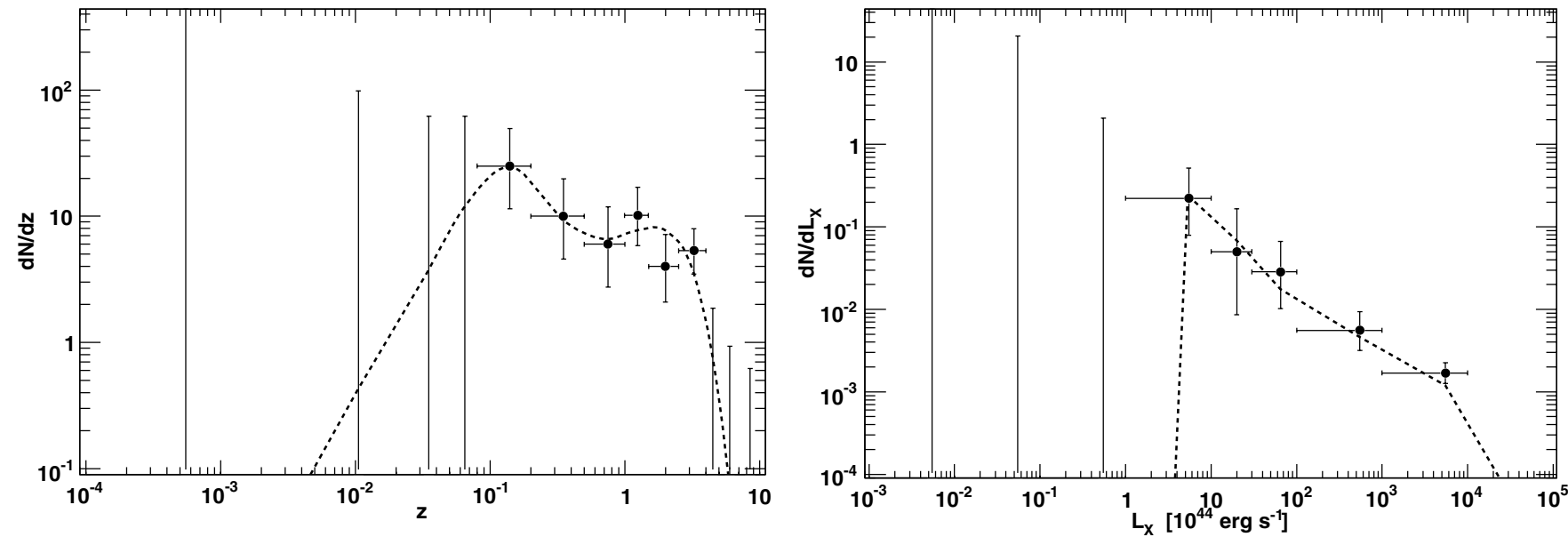

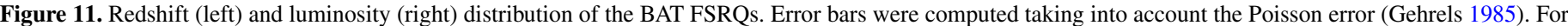
both cases, the dashed line represents the best-fit XLF model (model 10 in Table 4) convolved with the BAT sky coverage.
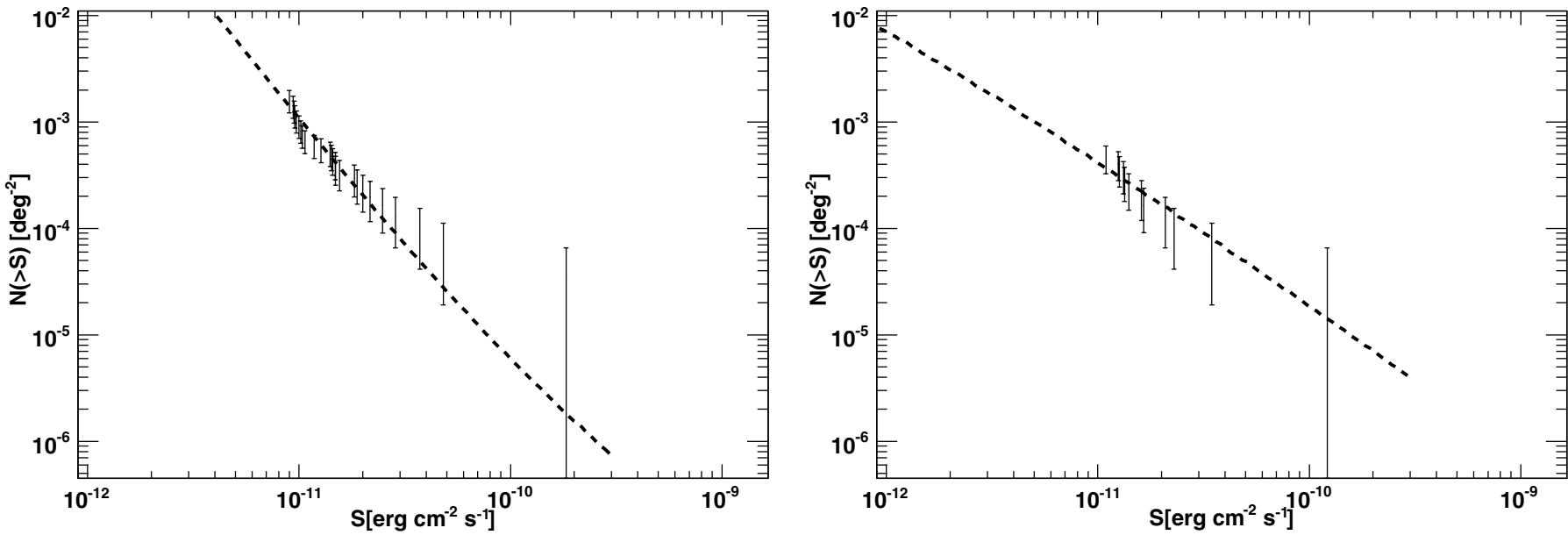

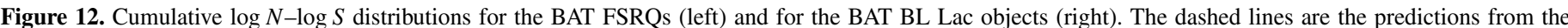

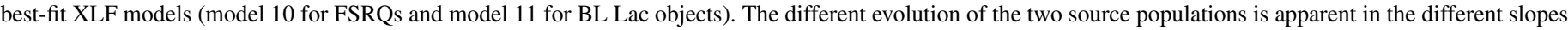
of the two $\log N-\log S$ distributions (steeper for FSRQs and flatter for BL Lac objects).
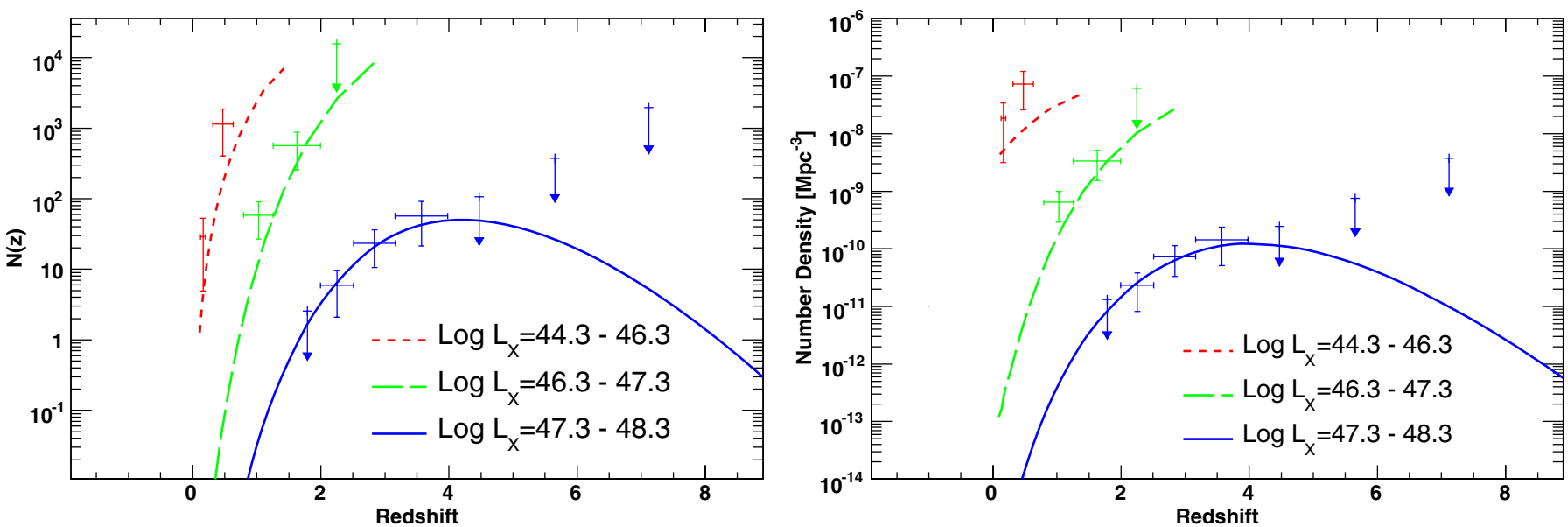

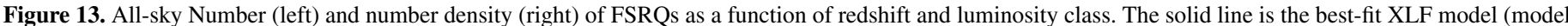
10 in Table 4). The BAT data (points with errors) were "deconvolved" taking into account the BAT sensitivity.

(A color version of this figure is available in the online journal.)

from the best fit. The BAT data were "deconvolved" with the method outlined in Section 4.2. It is clear that BAT is very effective in constraining the density of FSRQs at high luminosity and large redshifts. The same figure shows that the cutoff in the evolution is, at least for luminosities larger than $10^{47} \mathrm{erg} \mathrm{s}^{-1}$, well constrained. 

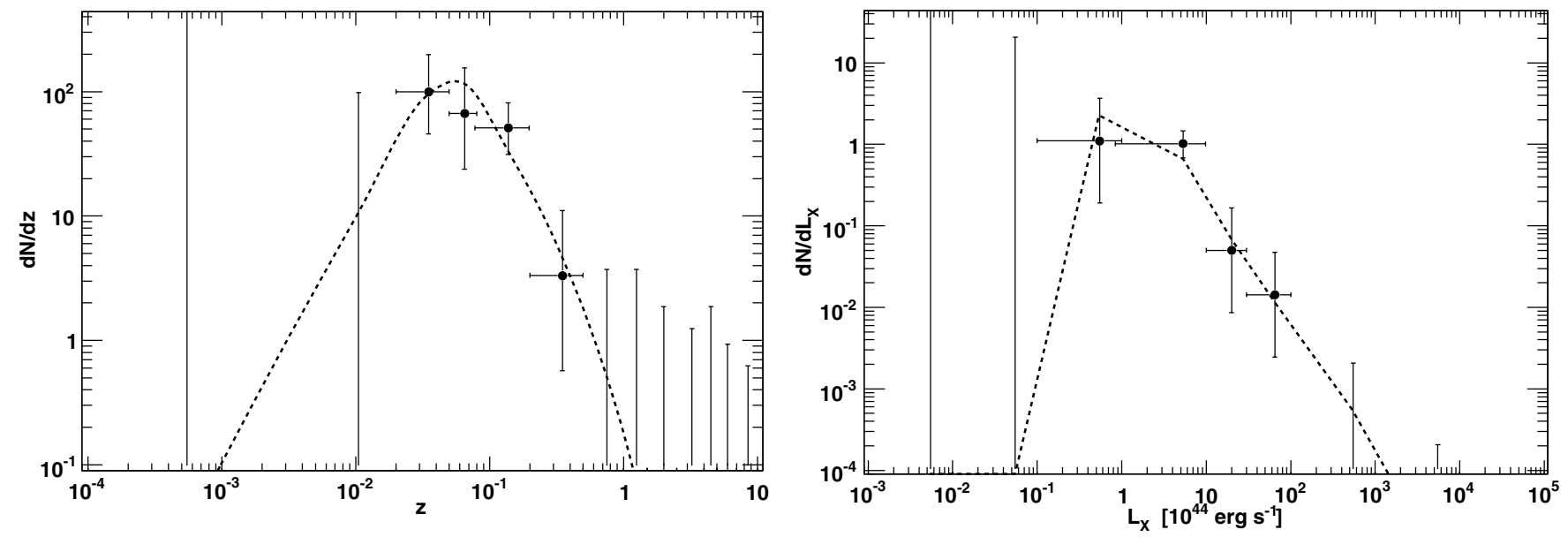

Figure 14. Redshift (left) and luminosity (right) distribution of the BAT BL Lac objects. Error bars were computed taking into account the Poisson error (Gehrels 1985). For both cases, the dashed line represents the PLE XLF (single power-law model, see model 11 in Table 4) convolved with the BAT sky coverage.

\subsubsection{BL Lac Objects}

Given the small number of BL Lac objects (12) and the relatively low redshift range that they span $(0.01<z<1.0)$ we cannot use complex evolutionary models. We thus tried to fit a simple PLE model to the data. We obtain an excellent fit which implies mild negative evolution (albeit with large errors). Indeed, the best-fit value of the evolutionary parameter is $-0.79 \pm 2.43$. As the results reported in Table 4 show, fixing the evolution parameter at zero produces an equally good fit (see models 11 and 12). Figure 14 shows that the best-fit XLF models (model 11) reproduces accurately the observed distributions in redshift and luminosity. The best-fit XLF predicts the cumulative source count distribution always within $1 \sigma$ (see the right panel of Figure 12). We also tried to use a double power-law model for the local XLF (see model 13). While this model reproduces the BAT data accurately, most of its parameters are poorly constrained. However, we note that the best-fit parameters are in good agreement with the values derived for the whole blazars sample (see model 7) and that the evolution, although still consistent with zero, became positive. Thus, we believe that given the small number of BL Lac objects it is currently impossible to constrain the sign of the evolution (i.e., positive or negative evolution).

\section{IMPLICATION FOR THE COSMIC X-RAY BACKGROUND}

Equation (17) can be used to estimate the contribution of blazars to the CXB outside the energy band of this survey. In this case, $F_{X}\left(L_{X}, z\right)=F_{X}\left(L_{X}, z, E\right)$ becomes a function of energy and represents the spectral energy distribution (SED) of the blazar source class. To model the contribution of FSRQs and BL Lac objects, we used the best-fit XLF models derived in the previous sections. These are model 9 and model 11, in Table 4, respectively, for FSRQs and BL Lac objects. We also remark that the results presented here do not change if other valid XLF models (e.g., model 10 for FSRQs and models 12 and 13 for BL Lac objects) are used. In order to compute correctly the uncertainties we employed a Monte Carlo simulation. We generated a large number $(>200)$ of luminosity functions starting from randomly sampled best-fit parameters drawn from the covariance matrix derived during the fit stage. Moreover, for each randomly generated XLF, a random photon index has been drawn from the index distributions of the given class (i.e., FSRQ or BL Lac). We then computed the contribution to the CXB for each of these luminosity functions and computed the $1 \sigma$ deviation, around the mean value, at given fixed energies. As a first test, we model the SED using a simple power-law model. Figure 15 shows the contributions of FSRQs and BL Lac objects (evolving as different populations) to the CXB. It is apparent that while the contribution of BL Lac objects appears negligible in this hypothesis (i.e., no BL Lac evolution), the contribution of FSRQs is substantial in hard X-rays. From our luminosity function, we derive that virtually $100 \%$ of the CXB for energies $>500 \mathrm{keV}$ is produced by FSRQs. We also use the synthesis model of Gilli et al. $(2007)^{12}$ to take into account the contribution of Seyferts to the CXB. We arbitrarily renormalize the Gilli et al. (2007) model by 1.1. This is justified by the fact that this synthesis model is tuned to reproduce the CXB as measured by HEAO-1 (Gruber et al. 1999) which is known to underestimate the CXB emission of $\sim 10 \%$ at $30 \mathrm{keV}$ (Ajello et al. 2008b, and references therein). It is apparent from Figure 15 that summing the contribution of blazars to the one of Seyferts achieves a good estimate of the intensity of the CXB emission up to the $\mathrm{MeV}$ range.

Modeling the SED with a simple power-law model is a straightforward and robust hypothesis, but it remains accurate only for extrapolations close to the original $15-55 \mathrm{keV}$ band. Indeed, Figure 15 shows that at $10 \mathrm{MeV}$ the contribution of FSRQs, computed in this way, overestimates the diffuse background by an order of magnitude. The $v F v$ spectrum of FSRQs exhibits an IC peak which is located somewhere in the $\mathrm{MeV}-\mathrm{GeV}$ band. While detailed modeling of the SEDs of each of the BAT blazars is outside the scope of this paper, we note that some of the BAT FSRQs were analyzed by several authors (Zhang et al. 2005; Sambruna et al. 2006, 2007; Tavecchio et al. 2007; Watanabe et al. 2009). In all cases, the authors find that the IC peak is located in the MeV band. We thus represent the SED with an empirical double power-law model of the type: $d N / d E \propto\left[\left(E / E_{b}\right)^{-\Gamma_{1}}+\left(E / E_{b}\right)^{-\Gamma_{2}}\right]^{-1}$, where $\Gamma_{1}$ and $\Gamma_{2}$ are the photon indices (1.6 and 2.5, respectively) before and after the energy break $E_{b}$. For the energy break, $E_{b}$, we chose a value of $1 \mathrm{MeV}$ motivated by the observations reported above. However, we note that the smooth and large curvature of the model we employ, makes it virtually insensitive to the exact value of $E_{b}$ if this is within 1 order of magnitude. Figure 16 shows the

\footnotetext{
12 A Web interface to the model of Gilli et al. (2007) is available at
} http://www.bo.astro.it/ gilli/xrb.html. 


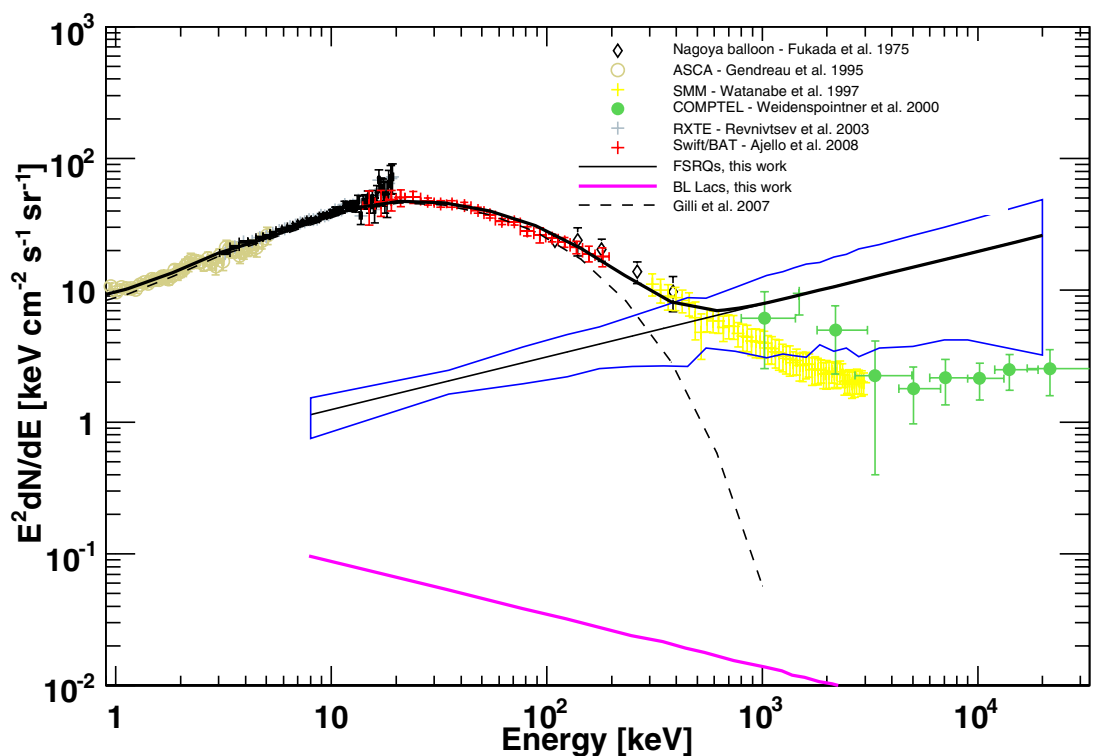

Figure 15. Spectrum of the CXB and contribution of the FSRQs (blue region). The data points are different measurements of the diffuse background as indicated in the label (Fukada et al. 1975; Gendreau et al. 1995; Watanabe et al. 1997; Weidenspointner et al. 2000; Revnivtsev et al. 2003; Ajello et al. 2008b). The dashed line is the total contribution of Seyfert-like AGNs computed with the model of Gilli et al. (2007) arbitrarily multiplied by 1.1 to fit the CXB emission at $30 \mathrm{keV}$. The solid line is the sum of the Seyfert-like and FSRQs. The spectrum of FSRQs has been modeled as a power-with a mean photon index of 1.6. The blue region represents the range of values obtained from the Monte Carlo realizations of best-fit parameter ranges. The magenta solid line represents the contribution of BL Lac objects whose uncertainty is not plotted for clarity, but is, due to the low number of objects, $>30 \%$ at any energy.

(A color version of this figure is available in the online journal.)

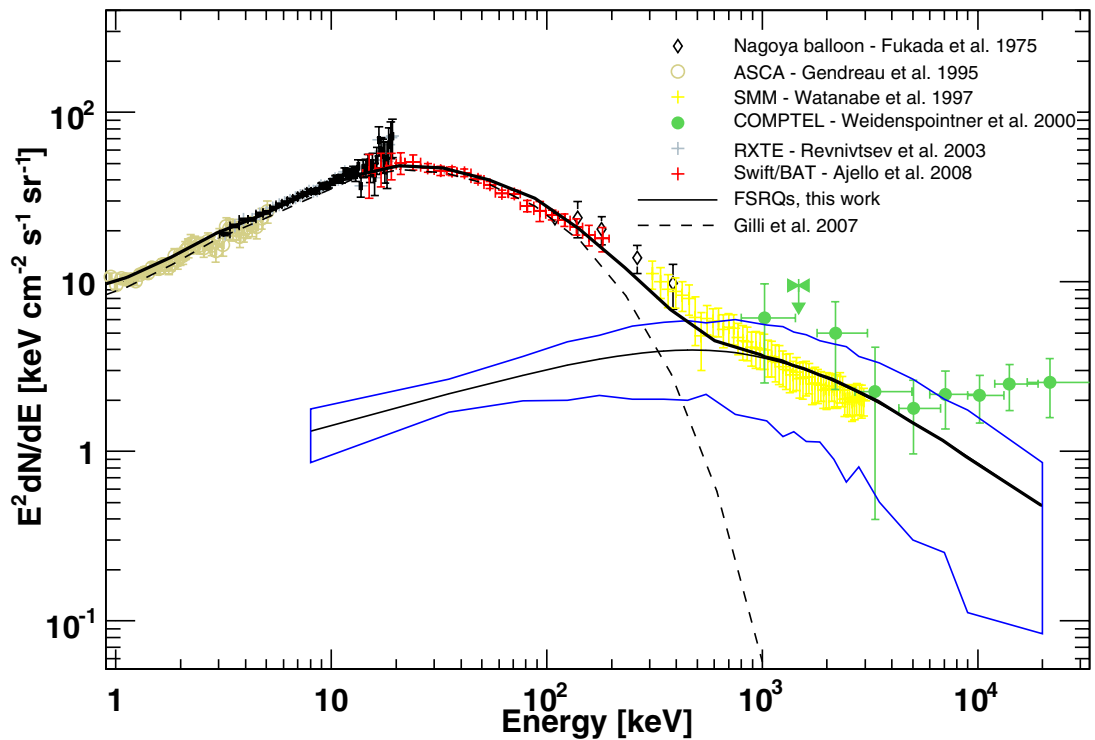

Figure 16. Contribution of FSRQs (blue region) to the CXB. The data are the same as in Figure 15, but in this case the SED of the FSRQs has been modeled with a double power-law function. The IC peak is located in the $\sim \mathrm{MeV}$ region. The contribution of BL Lac objects is the same as in Figure 15 and is not drawn here for clarity. The blue region represents the range of values obtained from the Monte Carlo realizations of best-fit parameter ranges.

(A color version of this figure is available in the online journal.)

contribution of FSRQs assuming that their IC peak is located in the $\mathrm{MeV}$ band. We find that in this case FSRQs account for the entire $\mathrm{CXB}$ emission up to $10 \mathrm{MeV}$. While there is basically no difference with respect to the single power-law case below $500 \mathrm{keV}$, the curvature of the IC peak makes the contribution of FSRQs to the CXB slightly smaller around $1 \mathrm{MeV}$. We also note that moving the IC peak beyond $10 \mathrm{MeV}$ produces a negligible curvature in the FSRQ integral emission and thus this case is well represented by the single power-law model.

Thus, the two analyses shown here cover well the case in which the IC peak is either located at $\mathrm{MeV}$ or at $\mathrm{GeV}$ energies (double and single power-law model, respectively). We must therefore conclude that the contribution of FSRQs to the diffuse emission is relevant and likely accounts for a substantial fraction (potentially $\sim 100 \%$ ) of the CXB around $1 \mathrm{MeV}$. Interpreting the $\mathrm{CXB}$ as a strong constraint, we derive that the population of FSRQ sampled by BAT must have the IC peak located in the $\mathrm{MeV}$ band in order not to overproduce the diffuse background at $\sim 10 \mathrm{MeV}$. Bhattacharya et al. (2009) recently reported for the FSRQs detected by EGRET a mean photon index of $2.34 \pm 0.15$. Since FSRQs have a mean photon index of 1.6 in BAT, this implies already that the IC peak is located 


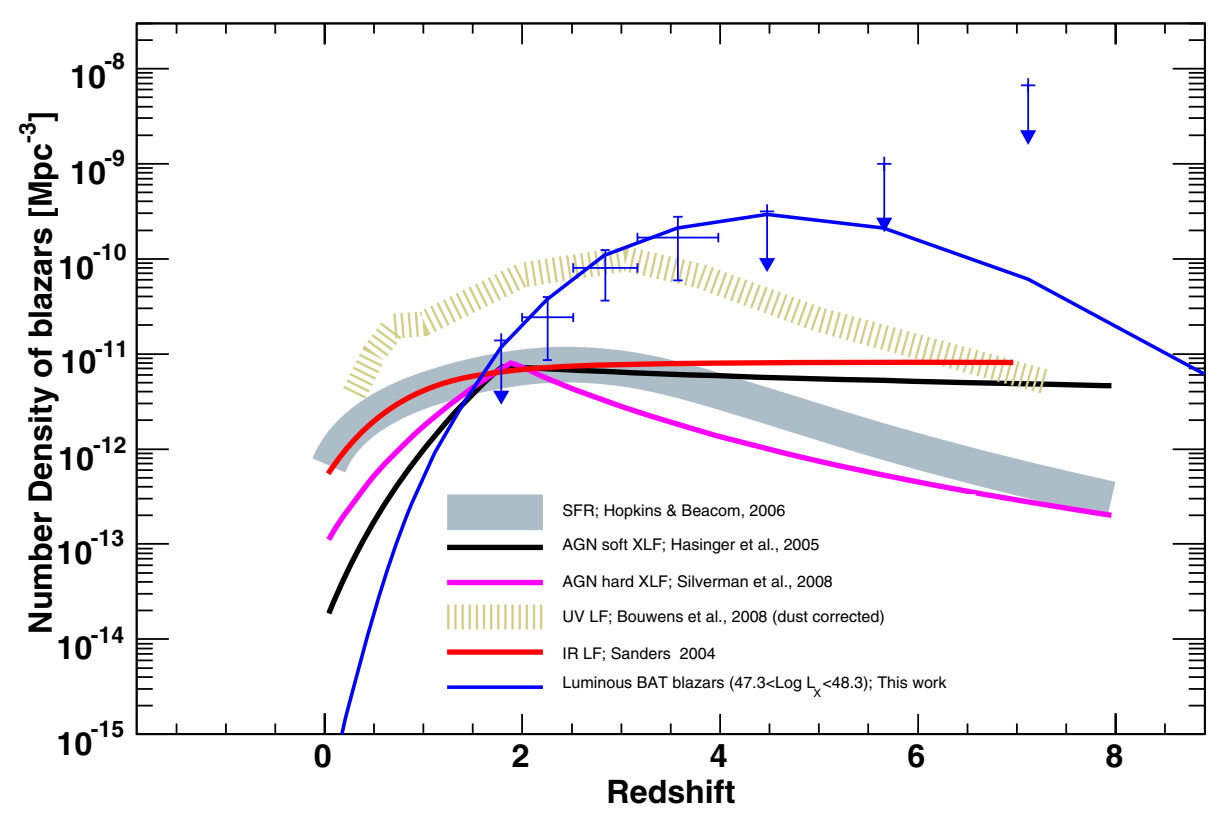

Figure 17. Density of the most luminous BAT blazars $\left(47.3<\log L_{X}<48.3\right.$, blue datapoints and line) as a function of redshift compared to: densities of AGNs from X-ray surveys (Hasinger et al. 2005; Silverman et al. 2008), star formation rate (Hopkins \& Beacom 2006) and densities of UV and IR galaxies (Bouwens et al. 2008; Sanders 2004, respectively). The different curves were rescaled arbitrarily to match the evolution of the BAT blazars. Thus, the comparison involves only the shape.

(A color version of this figure is available in the online journal.)

in between the BAT and EGRET energy bands. However, as we noted already in Section 2 only 9 FSRQs are in common between the EGRET and the BAT samples and this might imply that the other FSRQs detected by BAT have an IC peak at even lower energies. As it will be discussed in Section 7.2, FermiLAT will certainly clarify this scenario. Indeed, very recently, Abdo (2009a), discussing the results of the first three months of observations of Fermi-LAT, showed that FSRQs are detected by Fermi with a mean photon index of 2.4. Thus, FSRQs have soft spectra (photon index $>2.0$ ) in the $\mathrm{GeV}$ band, while they have hard spectra in the hard X-ray band. This confirms that their IC peak is located between the two bands. We note that the shape of the integrated emission of FSRQs is similar to the empirically derived one of Comastri et al. (2006).

We also found that the contribution of BL Lac objects is very small if they are a non-evolving (or mildly evolving) population. In agreement with Georgakakis et al. (2004), Galbiati et al. (2005), and Giommi et al. (2006), we find that the contribution of blazars (FSRQs and BL Lac objects) to the $2-10 \mathrm{keV} \mathrm{CXB}$ is $\sim 10 \%$.

\section{THE HIGH-REDSHIFT NON-THERMAL UNIVERSE}

The fact that the shape of the blazar luminosity function and its evolution are in agreement with those of X-ray-selected AGNs suggests the presence of a link between accretion and jet activity (e.g., Merloni et al. 2003). In other words, it seems that the most luminous AGNs (which in turn are the most luminous QSOs) harbor the most powerful blazars. This scenario takes place mostly in the very high-redshift universe where, thanks to the abundance of dust and gas, efficient accretion led to the build-up of massive QSOs. However, their space density quickly decreases, with cosmic time, leaving the room for the bulk of low-luminosity QSOs. This "antihierarchical" scenario, where larger structures come first, was also named "cosmological downsizing" (e.g., Cowie et al. 1999; Hasinger et al. 2005, and references therein) and constitutes a unique phenomenon which is not predicted in most of the semianalytic models based on Cold Dark Matter structure formation (Kauffmann \& Haehnelt 2000; Wyithe \& Loeb 2003). The late evolution of low-luminosity AGNs coincides well with the peak of the star formation in the universe (e.g., Hopkins \& Beacom 2006) highlighting once more the interconnection between the host and its nucleus. A mechanism of accretion with different efficiencies, as a function of cosmic time, has been invoked to explain the antihierarchical growth of AGNs (e.g., Merloni 2004). However, the fate of the very first and luminous quasars (i.e., the apparent disappearance of quasar activity in massive galaxies at late times) remains still unknown and there are doubts whether these objects can form at all in a $\Lambda$ CDM universe (Springel et al. 2005).

In Figure 17, we compare the shape of the evolution of the most luminous BAT blazars with: (1) the evolution of luminous X-ray-selected AGNs (Hasinger et al. 2005; Silverman et al. 2008), (2) the star formation history of the universe (Hopkins \& Beacom 2006), (3) and the evolution of UV and IR galaxies (Bouwens et al. 2008; Sanders 2004, respectively). The most direct comparison is clearly with AGNs. We note that the shape of the evolution is very similar, but that the cutoff in the AGNs growth is at lower redshifts $(z<2)$ with respect to the cutoff of BAT blazars. This means that the peak of the evolution of the BAT objects is at much earlier times in the history of the universe than the peak of the most luminous AGNs detected in the deepest X-ray surveys. The star formation history of the universe shows a similar trend and a peak around $z=\sim 2$ as for normal AGNs and this has been interpreted as the evidence of the strong link between AGNs and the star formation in its host galaxy. Assuming that the activity of the BAT blazars is powered by accretion onto supermassive black hole, this implies that the Doppler-boosting allows BAT to detect a class of objects (in luminosity) which escaped even the deepest X-ray surveys.

The only objects which show a peak in the evolution at redshift $>2$ are bright star-forming galaxies detected up to very high redshifts in the GOODS fields (Wall et al. 2008; Bouwens et al. 


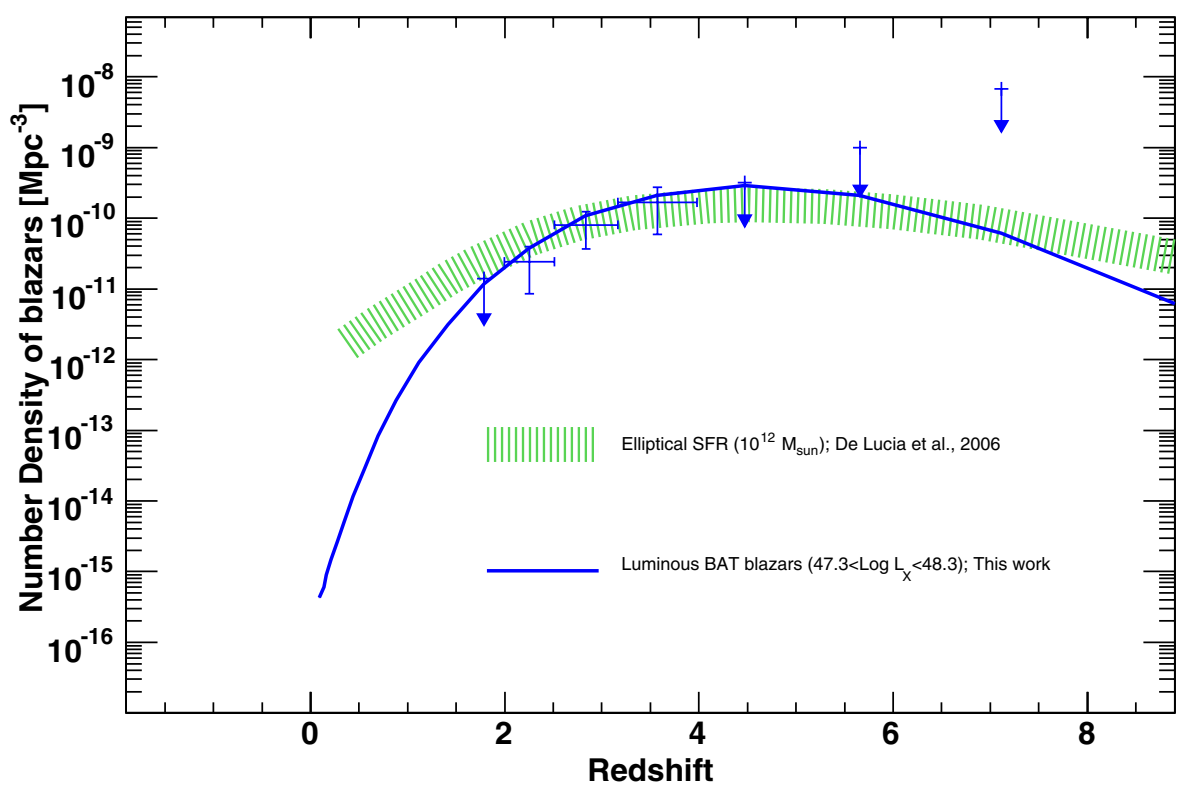

Figure 18. Density of the most luminous BAT blazars $\left(47.3<\log L_{X}<48.3\right.$, blue datapoints and line) compared to the prediction of the evolution of massive elliptical galaxies of De Lucia et al. (2006). The curve of De Lucia et al. (2006) was rescaled to match the BAT luminosity function. In order to consider also the contribution of misaligned jets, the space density of blazars ( $y$-axis) needs to be multiplied by a factor in the 200-800 range (see Section 6 for details).

(A color version of this figure is available in the online journal.)

2008 , and references therein). The rapid brightening of galaxies within the first two billion years is tightly connected to the assembly of large dark matter haloes (e.g., Wang \& Kauffmann 2008). An intriguing idea is that black holes are formed and fueled, and AGN activity is triggered during major galaxy mergers (Kauffmann \& Haehnelt 2000; Wyithe \& Loeb 2003; Croton et al. 2006). Although this constitutes a plausible fueling mechanism, the intermediate-luminosity AGNs are harbored by galaxies which display little or no merger events (Hasan 2007). However, to form a supermassive black hole, a more violent process such as a major merger event may be required to funnel a large amount of gas into the central region of the galaxy. It seems that the most massive galaxies undergo a major merger event at earlier times, and within the first 2-4 billion years, than less massive galaxies (Wang \& Kauffmann 2008; Stewart et al. 2008). If the bulk of the black hole mass is formed in this way, then it would explain the lack of growth of powerful AGNs at present times. The abundance of luminous blazars at large redshift fits well in this scenario since blazars are found in giant elliptical galaxies which in turn are supposed to have undergone a major merger event (Toomre \& Toomre 1972; Negroponte \& White 1983; Wang \& Kauffmann 2008). Thus, we argue that the luminous blazars can be used as a tracer of massive galaxies and merging activity in the very early universe.

Understanding the formation of massive galaxies is an important astrophysical issue because as much as $50 \%$ of the stellar mass in the local universe appears to be in early-type systems (Bell et al. 2003). The most massive galaxies (i.e., $\left.M_{*}>10^{11.5} M_{\odot}\right)$ appear to be already in place at $z \sim 2$ suggesting that they formed in the very early universe (see Conselice 2008, for a review). Unfortunately, present surveys, both in the optical and in X-rays, are not sensitive enough to make a statistical census beyond redshift 2. Here, BAT and more in general blazar surveys, can play an important role. In Figure 18, we compare the redshift evolution of the luminous BAT blazars with the prediction of the evolution of massive elliptical galaxies as determined, using simulations, by De Lucia et al. (2006). The similarity between the two curves is apparent and reinforces our idea that blazars can be used to study the formation of massive systems in the early universe.

The number density of massive ellipticals hosting active blazars is larger than the one reported in Figure 18 since we consider only those blazars pointing at us. Since there should be no difference between the population of blazars pointing at us and those pointing in all the other directions we might try to estimate their total number. The number of misaligned blazars, can be estimated as $N_{\text {TOT }} \approx 2 \Gamma^{2}$, where $\Gamma$ is the bulk Lorentz factor (e.g., Scheuer \& Readhead 1979). Assuming a standard value of $\Gamma=10$ (20) (see, e.g., Urry \& Padovani 1995; Sambruna et al. 2007) leads to a space density of ellipticals, hosting active blazars, which is 200 (800) times larger than that one shown in Figure 18 and thus of the order of 2.0 $8.0 \times 10^{-8} \mathrm{Mpc}^{-3}$ at a redshift of $\sim 4$.

Moreover, the excellent agreement between the evolution of massive galaxies and blazars suggests that there should be no intrinsic difference, at least in the early universe, between ellipticals and ellipticals hosting active blazars. This means that the fraction of ellipticals which host active blazars is not changing dramatically as a function of cosmic time.

Recently, Sikora et al. (2007) showed that on a radioloudness/Eddington-ratio diagram ellipticals and spirals form two distinct and well separated parallel sequences (see also Wilson \& Colbert 1995). They argue that this different behavior might be given by the spin. Indeed, within the hierarchical cosmological framework, the main difference in the evolution of giants ellipticals and spirals is that the first ones underwent at least one major merger event in the past. These events can produce a maximally rotating black hole by coalescence of the two black holes (Escala et al. 2004, 2005; Dotti et al. 2007) and by triggering large-gas accretion events that spin up the hole (Barnes \& Hernquist 1996; Escala 2007; Bogdanović et al. 2007). It is important to note that under the 
assumption that jets are powered by rotating black holes via the Blandford \& Znajek (1977) mechanism, the efficiency of the jet production is determined by the black hole spin. We believe that gathering a large sample of blazars which spans adequately luminosity and redshift will allow in detail to understand the role of spin and accretion in triggering jet activity. In this respect, BAT and, in particular, Fermi will play an important role.

\section{SUMMARY AND CONCLUSIONS}

\subsection{The Blazar XLF}

We have used a complete sample of blazars detected by BAT to derive the first luminosity function of blazars in the $15-55 \mathrm{keV}$ band. We have shown using several methods that BAT blazars are evolving strongly, while the Seyfert-like AGNs detected by BAT are not. The evolution of the blazars is implicit in the fact that BAT detects 10 objects at redshifts larger than 2.0. The local luminosity function (e.g., $\Phi\left(L_{X}, z=0\right)$ ) is compatible with a double power-law model where the faint-end slope is required to be flat mainly by the absence of low-luminosity BAT blazars. According to Urry \& Shafer (1984), the flattening of the local XLF at low luminosities might be produced by beaming which boosts intrinsically low-luminosity sources to high luminosities. The best-fit XLF models imply an evolution in luminosity which is epoch dependent (e.g., the evolution parameter changes with redshift) as was found for other samples of blazars or FSRQs (e.g., Wall 2008; Padovani et al. 2007). In all cases, it appears that blazars are evolving strongly up to a redshift cutoff which is, at least for the most luminous objects, well constrained by our data.

We find that the strong evolution of blazars is driven by the evolution of FSRQs which are the only objects detected by BAT at large redshifts. Our best-fit XLF, to the sample of BL Lac objects, shows that BL Lac objects have a negligible evolution. This is found to be in agreement with the results of Rector et al. (2000), Padovani et al. (2007), and Bhattacharya et al. (2009). Given the small number of objects in our sample we cannot rule out, nor confirm, the claims of negative evolution of BL Lac objects (e.g., Wolter et al. 1991; Beckmann et al. 2003). These last two samples reach a flux which is lower than the current BAT sensitivity, thus with a few more years of exposure BAT might be able to test this negative-evolution scenario. Finally, we also remark that given the small number of objects (12), the evolution of BL Lac objects is marginally consistent (at 1.5 $\sigma$ ) with the evolution of the FSRQ class.

The $\log N-\log S$ distribution of blazars is steeper than the one of Seyfert-like AGNs. Its slope of 1.9 is larger than the Euclidean value of 1.5 which characterizes the Seyfert galaxies (see Table 3 for details). We, thus, expect that the fraction of blazars will steadily increase among the total AGN population detected by BAT. We expect that in a relative short timescale (e.g., a couple of years) and, depending also on the systematic errors of the BAT survey, the blazar sample might contain more than 60 objects. This will be very important as it will allow us to improve the results and the prediction of the XLF models for both FSRQ and BL Lac objects. As we have shown, blazars are extremely rare objects which can be detected in hard X-rays only through large-area sky surveys. Our best-fit XLF model predicts a flattening in the $\log N-\log S$ at fluxes lower than $5 \times 10^{-13} \mathrm{erg} \mathrm{cm}^{-2} \mathrm{~s}^{-1}$. Thus, a mission like EXIST (Grindlay 2005 ) would detect FSRQs with a surface density of $\sim 0.5 \mathrm{deg}^{-2}$ at fluxes of $5 \times 10^{-13} \mathrm{erg} \mathrm{cm}^{-2} \mathrm{~s}^{-1}$. If confirmed that most of the BAT FRSQs are MeV blazars (see Sections 5 and 7.2), then an optimum band to select and study them would be the MeV band. A mission like GRIPS (Greiner et al. 2008) would gather a fairly large sample (500-1000) of blazars.

The redshift distribution of the BAT blazars (e.g., Figure 8) shows a peak at low redshift and a flat tail extending up to $z \approx 4$. This distribution differs from the redshift distributions of radio-selected blazars which display a peak at $z \approx 1.0$ 1.5 (e.g., Dunlop \& Peacock 1990; Wall et al. 2005). The reasons for this difference lie in the different selection effects and sensitivity of these surveys. Since BAT is sensitive only to bright X-ray fluxes most of the low-luminosity low-redshift sources are currently undetected. Additionally, the discrepancy in the redshift distributions between radio and hard X-rays might also be due to the different shapes of the evolution and of the local luminosity functions in these bands. This would not be surprising in view of the fact that radio and $\mathrm{X}$-rays probe different scales in these systems. A larger data set of hard X-ray-selected blazars will allow us to test these hypotheses.

From our XLF, we derive that the density of FSRQs at fluxes of $10^{-13} \mathrm{erg} \mathrm{cm}^{-2} \mathrm{~s}^{-1}$ is $5.2_{-3.7}^{+12.1} \mathrm{deg}^{-2}$ which is compatible with previous estimates in other X-ray bands (Wolter \& Celotti 2001; Giommi \& Colafrancesco 2006). Within the large uncertainties of our estimate, we derive that at faint fluxes the density of FSRQs is not negligible when compared to the total AGN population. As an example, the density of all AGNs in the XMMCOSMOS field is $24.0 \pm 3 \mathrm{deg}^{-2}$ for equivalent fluxes as above (Cappelluti et al. 2007). This means that deep X-ray surveys necessarily contain a $\sim 10 \%$ fraction of blazars. This seems in agreement with the finding of della Ceca et al. (1994) who report a fraction of radio-loud objects (among X-ray-selected AGNs) of $\sim 10 \%$ at fluxes of $10^{-13} \mathrm{erg} \mathrm{cm}^{-2} \mathrm{~s}^{-1}$. Similar fractions of radio-loud AGNs were also found by Hooper et al. (1996) and Zickgraf et al. (2003).

The main point of concern is however the selection of very absorbed, Compton-thick, AGNs in deep X-ray fields. Generally, given the lack of sufficient signal, the source intrinsic absorption is derived by an hardness-ratio analysis (e.g., Fiore et al. 2008; Brusa et al. 2009). The current "paradigm" is that exceptionally hard X-ray spectra (photon indices of 1.0-1.5) are likely produced by strong absorption. Our analysis shows that FSRQs have intrinsically hard X-ray spectra with photon indices sometimes lower than 1.6 (see Figure 2, but also Tavecchio et al. 2007; Watanabe et al. 2009). We believe that, if radio properties are not properly taken into account, selection of absorbed sources based solely on hardness ratios will produce a sample which can be contaminated by a substantial fraction of FSRQs.

\subsection{The Cosmic X-ray Background}

The origin of the MeV background has been a long-standing issue in astrophysics. Several astrophysical processes have been put forward to explain it. Among them, dark matter annihilation (Ahn \& Komatsu 2005), nuclear decays from SNe Ia (Clayton $\&$ Ward 1975), and nonthermal emission from Seyfert galaxies (Inoue et al. 2008) were the most important ones. We used our best-fit XLF model to make a prediction of the integrated emission due to FSRQs and derived that FSRQs account for most of the diffuse background emission for energies $>500 \mathrm{keV}$. Moreover, assuming that most of the FSRQs have an IC peak in the $\mathrm{MeV}$ band, as some of the BAT blazars (e.g., Zhang et al. 2005; Sambruna et al. 2007; Tavecchio et al. 2007; Watanabe et al. 2009), we showed that the sum of the contribution of 
emission-line AGNs (Gilli et al. 2007) and blazars reproduces well the CXB emission from $1 \mathrm{keV}$ to $10 \mathrm{MeV}$. Our prediction of the contribution of blazars to the CXB is well in agreement, in the $2-10 \mathrm{keV}$ band, with the findings of several authors (e.g., Galbiati et al. 2005; Comastri et al. 2006; Giommi et al. 2006).

Recently, Inoue et al. (2008) proposed that a population of nonthermal electrons present in the hot AGN coronae can account for a substantial part of the $\mathrm{MeV}$ background. Our finding shows that the nonthermal contribution from AGN coronae should be small as most of the diffuse background emission is accounted for by blazars. In a more recent work, Inoue \& Totani (2008) derived the luminosity function of EGRET blazars taking into account the blazar sequence; this is then used to compute the contribution of blazars to the diffuse background. From their best-fit model, it arises that blazars contribute negligible emission around $10 \mathrm{keV}$. This is in conflict with the main finding of this paper that blazars contribute $\sim 10 \%-20 \%{ }^{13}$ of the CXB emission in the $15-55 \mathrm{keV}$ band. As a matter of fact, $\sim 17 \%$ of all BAT AGNs are blazars and thus their contribution to the CXB must be of the same order.

The scenario which we derive from our data can be easily tested by the Fermi-LAT. Indeed, we showed that in order not to overproduce the MeV background, most FSRQs are required to "peak" at $\mathrm{MeV}$ energies for a large fraction of their time. Thus, the detection by LAT of soft FSRQs (e.g., photon indices of 2.2-2.5) would constitute a final evidence that the IC peak should be between the BAT and the LAT energy bands. On the other hand, the detection of hard FSRQs (indices of 1.4-1.8) would invalidate our prediction. The first Fermi-LAT results convalidate our results (Abdo 2009a). Indeed, FSRQs are detected by Fermi with a mean photon index of 2.4 (and a tail extending up to 3.0) confirming that the IC peak must be located somewhere between the $\mathrm{keV}$ and the $\mathrm{GeV}$ band.

\subsection{Tracing the Star Formation History of Massive Ellipticals at High Redshift}

The similar evolution of radio-quiet AGNs and star formation history of normal galaxies has been interpreted as the evidence of the co-evolution of AGNs and their hosts (e.g., Madau et al. 1996; Hasinger et al. 2005).

The main, serendipitous, finding of our analysis is that the evolution of the BAT blazars shows a redshift cutoff which is larger than previously found for other, mostly radio-quiet, AGN samples. This is found to be $z_{c}=4.3 \pm 0.5$ for blazars of typical luminosities exceeding $10^{47} \mathrm{erg} \mathrm{s}^{-1}$. The large redshift cutoff shows that the most luminous blazars formed very early in the universe and then their number density quickly decreased. To our knowledge, no other source class displays a similar extreme evolution. X-ray surveys show that the redshift cutoff increases with luminosity (Ueda et al. 2003; Hasinger et al. 2005; La Franca et al. 2005; Silverman et al. 2008) and thus we believe that Doppler boosting, due to the relativistic beaming, allows BAT to detect rare objects which escaped even the deepest surveys.

We compared the blazars luminosity function and the prediction of the star formation history of massive elliptical galaxies (De Lucia et al. 2006) and found good agreement. However, this

\footnotetext{
13 The BAT blazar XLF's main uncertainty is given by the small number of objects. Moreover, the CXB fraction increases if FSRQs and BL Lac objects are treated as a single population.
}

agreement is not entirely surprising if one realizes that blazars are normally found in giant elliptical galaxies (Urry \& Padovani 1995; Falomo et al. 2000; O'Dowd et al. 2002). This represents another evidence that AGNs (jet activity in this case) and their hosts co-evolve through the history of the universe. However, tracing the evolution of giant galaxies is currently at the limit, or beyond, of the present-generation instruments and thus the use of blazars might represent the only approach to understand the formation of the most massive galaxies in the early universe.

Elliptical galaxies are thought to be the only objects which undergo one major merger (e.g., Wang \& Kauffmann 2008), and in particular this seems to happen in the first billion years of the universe. As a natural consequence, it is believed that merging activity would produce a rapidly spinning black hole (e.g., Volonteri et al. 2007) which on theoretical grounds is required to explain the production of a collimated, relativistic, outflow (Blandford \& Znajek 1977; Blandford et al. 1990). Sikora et al. (2007) found out that on a radio-loudness/Eddingtonratio diagram elliptical and disk/spiral galaxies form different sequences and invoke the spin as the black hole parameter which might explain this different behavior. Larger blazar samples, better understanding of the evolution of massive systems, and direct black hole spin measurements will help in clarifying the jet-spin-merger scenario.

It is a pleasure to thank the referee of his/her comments which improved the paper. M.A. acknowledges very helpful suggestions from A. Comastri, R. Gilli, and A. Reimer and interesting discussions with A. Tramacere about blazars (and his guidance during the first Fermi flare-advocate duty shift). The help of D. Burlon with XMM-Newton data is acknowledged. This research has made use of the NASA/IPAC extragalactic Database (NED) which is operated by the Jet Propulsion Laboratory, of data obtained from the High Energy Astrophysics Science Archive Research Center (HEASARC) provided by NASA's Goddard Space Flight Center, and of the SIMBAD Astronomical Database which is operated by the Centre de Données astronomiques de Strasbourg.

Facilities: Swift/BAT.

\section{APPENDIX}

\section{THE SEYFERT SAMPLE}

In this appendix, we report the 199 Seyfert objects which are used as a control sample plus the six radio galaxies detected by BAT. The sample, shown in Table 5, is reported here as a reference for the reader to demonstrate that the classifications reported in Table 2 are accurate. We note that 152 out of the 205 sources reported in this table are also detected in the BAT 22 month survey of Tueller et al. (2009). The main differences among the two analyses are as follows.

1. The different energy band used (15-55 keV band versus the 15-195 keV band adopted by Tueller et al. 2009).

2. The different exposure used (36 months versus the 22 months used by Tueller et al. 2009).

3 . The slightly different data filtering and screening techniques (see Ajello et al. 2008a; Tueller et al. 2009, for details).

Despite these differences, $\sim 75 \%$ of the sources detected in this analysis are also contained in the sample of Tueller et al. (2009). 
Table 5

Sample of Seyferts and Radio Galaxies

\begin{tabular}{|c|c|c|c|c|c|c|c|c|}
\hline SWIFT NAME & $\begin{array}{c}\text { R.A. } \\
\text { (J2000) }\end{array}$ & $\begin{array}{c}\text { Decl. } \\
(\mathrm{J} 2000)\end{array}$ & $\begin{array}{c}\text { Flux } \\
\left(10^{-11} \mathrm{cgs}\right)\end{array}$ & $\mathrm{S} / \mathrm{N}$ & $\mathrm{ID}^{\mathrm{a}}$ & Type $^{b}$ & Redshift & In BAT 22 months ${ }^{\mathrm{c}}$ ? \\
\hline $\mathrm{J} 0006.4+2009$ & 1.600 & 20.152 & $1.16 \pm 0.20$ & 5.8 & Mrk 335 & Sy1 & 0.0254 & $\mathrm{y}$ \\
\hline J0038.6+2336 & 9.650 & 23.600 & $1.10 \pm 0.21$ & 5.3 & Mrk 344 & Sy & 0.0240 & \\
\hline J0042.7-2332 & 10.680 & -23.548 & $2.44 \pm 0.21$ & 11.7 & NGC 235A & Sy2 & 0.0222 & $\mathrm{y}$ \\
\hline J0048.7+3157 & 12.188 & 31.962 & $7.71 \pm 0.20$ & 37.8 & Mrk 348 & Sy2 & 0.0150 & $\mathrm{y}$ \\
\hline J0051.9+1726 & 12.998 & 17.447 & $1.81 \pm 0.21$ & 8.6 & QSO B0049+171 & Sy 1 & 0.0642 & $\mathrm{y}$ \\
\hline J0059.9+3149 & 14.997 & 31.831 & $1.66 \pm 0.21$ & 8.0 & SWIFT J0059.4+3150 & Sy1 & 0.0149 & $\mathrm{y}$ \\
\hline J0101.0-4748 & 15.274 & -47.800 & $0.97 \pm 0.18$ & 5.6 & 2MASX J01003469-478303 & GALAXY & 0.0753 & y \\
\hline J0108.8+1321 & 17.201 & 13.351 & $1.78 \pm 0.22$ & 8.2 & $4 \mathrm{C} 13.07$ & Sy2 & 0.0596 & $\mathrm{y}$ \\
\hline J0111.4-3805 & 17.867 & -38.086 & $1.52 \pm 0.18$ & 8.3 & NGC 424 & Sy2 & 0.0116 & \\
\hline J0113.8-1450 & 18.453 & -14.850 & $1.24 \pm 0.21$ & 5.8 & Mrk 1152 & Sy1 & 0.0522 & $\mathrm{y}$ \\
\hline J0114.3-5524 & 18.600 & -55.400 & $0.92 \pm 0.17$ & 5.3 & SWIFT J0114.4-5522 & Sy2 & 0.0121 & \\
\hline J0123.8-5847 & 20.952 & -58.785 & $2.65 \pm 0.17$ & 15.3 & Fairall 9 & Sy1 & 0.0470 & $\mathrm{y}$ \\
\hline J0123.8-3504 & 20.974 & -35.067 & $2.72 \pm 0.18$ & 14.7 & NGC 526A & Sy 1.5 & 0.0191 & y \\
\hline J0127.9-1850 & 22.000 & -18.847 & $1.27 \pm 0.20$ & 6.2 & MCG-03-04-072 & Sy1 & 0.0430 & \\
\hline J0134.0-3629 & 23.506 & -36.486 & $2.36 \pm 0.18$ & 13.0 & NGC 612 & GALAXY & 0.0298 & $\mathrm{y}$ \\
\hline J0138.6-4000 & 24.674 & -40.008 & $3.17 \pm 0.18$ & 18.0 & ESO 297-018 & Sy 2 & 0.0252 & $\mathrm{y}$ \\
\hline J0142.6+0118 & 25.652 & 1.300 & $1.28 \pm 0.22$ & 5.7 & [VV2003c] J014214.0+011615 & Sy 1 & 0.0500 & \\
\hline J0152.9-0326 & 28.250 & -3.448 & $1.47 \pm 0.22$ & 6.6 & IGR J01528-0326 & Sy2 & 0.0172 & $\mathrm{y}$ \\
\hline J0201.2-0649 & 30.320 & -6.821 & $4.17 \pm 0.22$ & 19.3 & NGC 788 & Sy2 & 0.0136 & $\mathrm{y}$ \\
\hline J0206.5-0016 & 31.631 & -0.270 & $1.53 \pm 0.22$ & 6.9 & MRK 1018 & Sy1.5 & 0.0424 & y \\
\hline J0215.0-0044 & 33.751 & -0.749 & $1.30 \pm 0.22$ & 5.9 & Mrk 590 & Sy1.2 & 0.0265 & \\
\hline J0226.0-6315 & 36.500 & -63.250 & $0.91 \pm 0.18$ & 5.2 & FAIRALL 0926 & Sy1 & 0.0580 & \\
\hline J0226.8-2819 & 36.703 & -28.324 & $1.14 \pm 0.18$ & 6.4 & 2MASX J02262568-2820588 & Sy 1 & 0.0600 & \\
\hline J0228.4+3118 & 37.120 & 31.316 & $4.38 \pm 0.23$ & 19.4 & NGC 931 & Sy1.5 & 0.0166 & $\mathrm{y}$ \\
\hline J0232.0-3639 & 38.020 & -36.662 & $1.09 \pm 0.17$ & 6.4 & IC 1816 & Sy2 & 0.0169 & $\mathrm{y}$ \\
\hline $\mathrm{J} 0234.4+3229$ & 38.612 & 32.489 & $1.60 \pm 0.23$ & 7.1 & NGC 973 & Sy2 & 0.0167 & $\mathrm{y}$ \\
\hline J0234.8-0847 & 38.702 & -8.794 & $2.13 \pm 0.21$ & 10.2 & NGC 985 & Sy 1 & 0.0430 & $\mathrm{y}$ \\
\hline J0235.6-2935 & 38.900 & -29.600 & $0.99 \pm 0.18$ & 5.6 & ESO 0416-G0002 & Sy 1.9 & 0.0592 & $\mathrm{y}$ \\
\hline J0238.5-5213 & 39.647 & -52.220 & $1.31 \pm 0.17$ & 7.6 & ESO 198-024 & Sy 1 & 0.0452 & $\mathrm{y}$ \\
\hline J0239.0-4043 & 39.767 & -40.732 & $0.97 \pm 0.17$ & 5.8 & 2MASX J02384897-4038377 & Sy1 & 0.0610 & \\
\hline J0241.5-0813 & 40.381 & -8.220 & $1.34 \pm 0.21$ & 6.4 & NGC 1052 & Sy2 & 0.0050 & $\mathrm{y}$ \\
\hline J0242.9-0000 & 40.732 & -0.012 & $2.00 \pm 0.22$ & 8.9 & NGC 1068 & Sy2 & 0.0038 & y \\
\hline J0249.3+2627 & 42.349 & 26.451 & $1.25 \pm 0.23$ & 5.5 & IRAS $02461+2618$ & GALAXY & 0.0580 & y \\
\hline J0252.8-0830 & 43.200 & -8.500 & $1.06 \pm 0.21$ & 5.0 & MCG-02-08-014 & Sy2 & 0.0168 & y \\
\hline J0255.4-0010 & 43.873 & -0.170 & $4.48 \pm 0.22$ & 20.1 & NGC 1142 & Sy2 & 0.0288 & y \\
\hline J0256.4-3212 & 44.117 & -32.208 & $1.31 \pm 0.17$ & 7.7 & ESO 417-6 & Sy2 & 0.0164 & y \\
\hline J0311.6-2045 & 47.919 & -20.760 & $1.27 \pm 0.18$ & 6.9 & 2MASX J03111883-2046184 & Sy 1 & 0.0660 & $\mathrm{y}$ \\
\hline $\mathrm{J} 0325.1+3409$ & 51.296 & 34.152 & $1.61 \pm 0.24$ & 6.8 & 2MASX J03244119+3410459 & Sy 1 & 0.0629 & $\mathrm{y}$ \\
\hline J0333.5+3716 & 53.397 & 37.278 & $1.63 \pm 0.24$ & 6.8 & IGR J03334+3718 & Sy 1 & 0.0574 & \\
\hline J0333.7-3608 & 53.433 & -36.141 & $3.12 \pm 0.17$ & 18.9 & NGC 1365 & Sy 1.8 & 0.0055 & y \\
\hline $\mathrm{J} 0342.2-2114$ & 55.554 & -21.244 & $2.15 \pm 0.18$ & 11.8 & SWIFT J0342.0-2115 & Sy1 & 0.0145 & $\mathrm{y}$ \\
\hline J0347.3-3029 & 56.850 & -30.500 & $0.89 \pm 0.17$ & 5.3 & RBS 0741 & Sy1 & 0.0950 & \\
\hline $\mathrm{J} 0350.7-5022$ & 57.679 & -50.377 & $1.29 \pm 0.17$ & 7.5 & SWIFT J0350.1-5019 & GALAXY & 0.0365 & $\mathrm{y}$ \\
\hline J0357.0-4039 & 59.268 & -40.666 & $0.89 \pm 0.17$ & 5.4 & 2MASX J03565655-4041453 & GALAXY & 0.0747 & \\
\hline J0402.5-1804 & 60.639 & -18.077 & $1.35 \pm 0.19$ & 7.0 & ESO 549- G049 & Sy2 & 0.0262 & $\mathrm{y}$ \\
\hline $\mathrm{J} 0407.5+0342$ & 61.883 & 3.717 & $1.90 \pm 0.25$ & 7.6 & $3 \mathrm{C} 105$ & Sy2 & 0.0890 & y \\
\hline J0415.2-0753 & 63.800 & -7.900 & $1.31 \pm 0.23$ & 5.6 & LEDA 14727 & Sy1 & 0.0379 & y \\
\hline J0426.4-5712 & 66.603 & -57.201 & $1.40 \pm 0.17$ & 8.2 & 1H 0419-577 & Sy 1 & 0.1040 & $\mathrm{y}$ \\
\hline J0433.4+0521 & 68.355 & 5.365 & $5.21 \pm 0.26$ & 19.8 & $3 \mathrm{C}-120$ & Sy 1 & 0.0330 & $\mathrm{y}$ \\
\hline J0438.5-1049 & 69.633 & -10.830 & $1.48 \pm 0.23$ & 6.4 & MCG-02-12-050 & Sy 1 & 0.0360 & $\mathrm{y}$ \\
\hline J0444.7-2812 & 71.199 & -28.200 & $1.07 \pm 0.18$ & 5.9 & 2MASX J04450628-2820284 & Sy2 & 0.1470 & \\
\hline J0451.8-5807 & 72.966 & -58.133 & $0.88 \pm 0.17$ & 5.2 & RBS 0594 & Sy 1 & 0.0900 & y \\
\hline J0453.5+0403 & 73.380 & 4.060 & $2.11 \pm 0.28$ & 7.6 & CGCG 420-015 & Sy2 & 0.0296 & $\mathrm{y}$ \\
\hline $\mathrm{J} 0455.3-7528$ & 73.841 & -75.477 & $1.27 \pm 0.18$ & 6.9 & ESO 33-2 & Sy2 & 0.0184 & $\mathrm{y}$ \\
\hline J0505.9-2351 & 76.497 & -23.854 & $2.78 \pm 0.20$ & 13.9 & XSS J05054-2348 & Sy2 & 0.0350 & $\mathrm{y}$ \\
\hline J0516.2-0009 & 79.071 & -0.161 & $4.11 \pm 0.28$ & 14.5 & QSO B0513-002 & Sy 1 & 0.0327 & y \\
\hline J0519.7-3240 & 79.930 & -32.676 & $2.38 \pm 0.19$ & 12.9 & SWIFT J0519.5-3140 & Sy2 & 0.0350 & $\mathrm{y}$ \\
\hline J0519.8-4546 & 79.963 & -45.774 & $2.49 \pm 0.17$ & 14.9 & Pictor-A & Sy1 & 0.0351 & y \\
\hline $\mathrm{J} 0524.2-1212$ & 81.050 & -12.200 & $1.42 \pm 0.25$ & 5.6 & LEDA 17233 & Sy1 & 0.0490 & $\mathrm{y}$ \\
\hline J0552.3-0727 & 88.090 & -7.457 & $14.75 \pm 0.29$ & 51.7 & NGC 2110 & Sy2 & 0.0078 & $\mathrm{y}$ \\
\hline J0552.3+5929 & 88.100 & 59.500 & $1.14 \pm 0.21$ & 5.3 & IRAS 05480+597 & Sy 1 & 0.0585 & \\
\hline J0558.1-3820 & 89.549 & -38.347 & $2.12 \pm 0.18$ & 11.6 & EXO 055620-3820.2 & Sy 1 & 0.0340 & $\mathrm{y}$ \\
\hline J0559.9-5026 & 89.980 & -50.441 & $0.94 \pm 0.17$ & 5.7 & PKS 0558-504 & RG & 0.1370 & \\
\hline J0602.9-8633 & 90.749 & -86.555 & $1.82 \pm 0.22$ & 8.4 & SWIFT J0601.9-8636 & Sy2 & 0.0064 & $\mathrm{y}$ \\
\hline
\end{tabular}


Table 5

(Continued)

\begin{tabular}{|c|c|c|c|c|c|c|c|c|}
\hline SWIFT NAME & $\begin{array}{c}\text { R.A. } \\
(\mathrm{J} 2000)\end{array}$ & $\begin{array}{c}\text { Decl. } \\
(\mathrm{J} 2000)\end{array}$ & $\begin{array}{c}\text { Flux } \\
\left(10^{-11} \mathrm{cgs}\right) \\
\end{array}$ & $\mathrm{S} / \mathrm{N}$ & $\mathrm{ID}^{\mathrm{a}}$ & Type $^{\mathrm{b}}$ & Redshift & In BAT 22 months ${ }^{\mathrm{c}}$ ? \\
\hline J0603.1+6523 & 90.799 & 65.399 & $1.38 \pm 0.20$ & 6.8 & UGC 3386 & GALAXY & 0.0154 & \\
\hline J0615.8+7101 & 93.967 & 71.021 & $6.08 \pm 0.20$ & 30.8 & Mrk 3 & Sy2 & 0.0135 & $\mathrm{y}$ \\
\hline J0623.9-3214 & 95.994 & -32.248 & $1.53 \pm 0.20$ & 7.5 & ESO 426-G 002 & GALAXY & 0.0224 & $\mathrm{y}$ \\
\hline J0624.1-6059 & 96.028 & -60.998 & $1.25 \pm 0.17$ & 7.4 & SWIFT J2141.0+1603 & Sy2 & 0.0410 & \\
\hline J0640.7-4324 & 100.200 & -43.400 & $0.92 \pm 0.18$ & 5.2 & 2MASX J06400609-4327591 & GALAXY & 0.0570 & $\mathrm{y}$ \\
\hline J0652.1+7425 & 103.044 & 74.425 & $3.29 \pm 0.19$ & 17.1 & Mrk 6 & Sy 1.5 & 0.0188 & $\mathrm{y}$ \\
\hline J0656.1+3959 & 104.027 & 39.986 & $2.29 \pm 0.26$ & 8.7 & UGC 3601 & Sy1 & 0.0172 & $\mathrm{y}$ \\
\hline J0718.0+4405 & 109.517 & 44.084 & $1.67 \pm 0.24$ & 7.1 & 2MASX J07180060+4405271 & Sy1 & 0.0610 & \\
\hline $\mathrm{J} 0742.5+4947$ & 115.644 & 49.793 & $2.98 \pm 0.21$ & 14.4 & Mrk 79 & Sy 1.2 & 0.0222 & $\mathrm{y}$ \\
\hline $\mathrm{J} 0800.1+2322$ & 120.032 & 23.370 & $1.62 \pm 0.24$ & 6.6 & SDSS J0759.87+232448.3 & GALAXY & 0.0290 & $\mathrm{y}$ \\
\hline $\mathrm{J} 0800.3+2638$ & 120.099 & 26.648 & $1.79 \pm 0.24$ & 7.5 & IC 486 & Sy1 & 0.0272 & $\mathrm{y}$ \\
\hline J0804.2+0506 & 121.050 & 5.101 & $3.18 \pm 0.25$ & 12.6 & UGC 4203 & Sy2 & 0.0135 & $\mathrm{y}$ \\
\hline J0811.1+7602 & 122.798 & 76.049 & $1.15 \pm 0.19$ & 6.2 & PG $0804+761$ & Sy 1 & 0.1000 & \\
\hline J0814.4+0423 & 123.600 & 4.400 & $1.26 \pm 0.24$ & 5.2 & CGCG 031-072 & Sy1 & 0.0331 & \\
\hline J0823.2-0456 & 125.800 & -4.947 & $1.35 \pm 0.23$ & 6.0 & SWIFT J0823.4-0457 & Sy2 & 0.0218 & $\mathrm{y}$ \\
\hline J0832.8+3706 & 128.200 & 37.100 & $1.03 \pm 0.20$ & 5.2 & RB 0707 & Sy 1.2 & 0.0919 & \\
\hline J0839.8-1214 & 129.950 & -12.248 & $1.28 \pm 0.21$ & 6.1 & $3 C 206$ & Sy1 & 0.1978 & $\mathrm{y}$ \\
\hline J0904.9+5537 & 136.250 & 55.632 & $1.04 \pm 0.17$ & 6.0 & SWIFT J0904.3+5538 & Sy1 & 0.0370 & $\mathrm{y}$ \\
\hline J0911.5+4528 & 137.898 & 45.471 & $1.23 \pm 0.18$ & 7.0 & SWIFT J0911.2+4533 & GALAXY & 0.0268 & $\mathrm{y}$ \\
\hline J0918.4+1618 & 139.615 & 16.316 & $1.65 \pm 0.21$ & 8.0 & Mrk 104 & Sy 1.5 & 0.0292 & $\mathrm{y}$ \\
\hline J0921.0-0803 & 140.257 & -8.067 & $2.59 \pm 0.20$ & 12.9 & SWIFT J0920.8-0805 & Sy2 & 0.0198 & $\mathrm{y}$ \\
\hline J0923.8+2256 & 140.962 & 22.936 & $2.05 \pm 0.20$ & 10.5 & MCG +04-22-042 & Sy 1.2 & 0.0327 & $\mathrm{y}$ \\
\hline J0925.2+5217 & 141.316 & 52.285 & $3.03 \pm 0.17$ & 18.1 & Mrk 110 & Sy1 & 0.0353 & $\mathrm{y}$ \\
\hline J0945.8-1419 & 146.468 & -14.332 & $1.28 \pm 0.21$ & 6.1 & NGC 2992 & Sy2 & 0.0077 & $\mathrm{y}$ \\
\hline J0947.7-3056 & 146.939 & -30.948 & $11.50 \pm 0.22$ & 51.5 & ESO 434-40 & Sy2 & 0.0085 & $\mathrm{y}$ \\
\hline J0947.9+0727 & 147.000 & 7.451 & $1.12 \pm 0.21$ & 5.4 & $3 C 227$ & RG & 0.0860 & $\mathrm{y}$ \\
\hline J0959.6-2250 & 149.916 & -22.834 & $4.44 \pm 0.22$ & 19.8 & NGC 3081 & Sy2 & 0.0080 & $\mathrm{y}$ \\
\hline $\mathrm{J} 1001.8+5542$ & 150.453 & 55.700 & $1.43 \pm 0.16$ & 8.8 & NGC 3079 & Sy2 & 0.0037 & $\mathrm{y}$ \\
\hline J1006.0-2306 & 151.500 & -23.100 & $1.25 \pm 0.23$ & 5.5 & ESO 499-G 041 & Sy1 & 0.0127 & \\
\hline $\mathrm{J} 1021.7-0327$ & 155.450 & -3.450 & $1.35 \pm 0.22$ & 6.3 & $\mathrm{MCG}+00-27-002$ & Sy1 & 0.0409 & \\
\hline $\mathrm{J} 1023.5+1951$ & 155.888 & 19.864 & $7.35 \pm 0.20$ & 36.8 & NGC 3227 & Sy 1.5 & 0.0039 & $\mathrm{y}$ \\
\hline $\mathrm{J} 1031.8-3451$ & 157.975 & -34.860 & $4.71 \pm 0.26$ & 18.4 & NGC 3281 & Sy2 & 0.0107 & $\mathrm{y}$ \\
\hline J1031.9-1417 & 157.996 & -14.300 & $2.13 \pm 0.23$ & 9.3 & H $1029-140$ & Sy1 & 0.0860 & \\
\hline $\mathrm{J} 1044.0+7023$ & 161.003 & 70.400 & $0.99 \pm 0.17$ & 5.9 & MCG+12-10-067 & Sy2 & 0.0333 & \\
\hline $\mathrm{J} 1046.5+2556$ & 161.649 & 25.950 & $1.12 \pm 0.19$ & 5.9 & UGC 05881 & GALAXY & 0.0200 & \\
\hline $\mathrm{J} 1048.5-2512$ & 162.149 & -25.200 & $1.42 \pm 0.27$ & 5.3 & NGC 3393 & Sy2 & 0.0125 & \\
\hline $\mathrm{J} 1049.3+2256$ & 162.350 & 22.950 & $1.57 \pm 0.20$ & 8.0 & SWIFT J1049.4+2258 & Sy2 & 0.0328 & $\mathrm{y}$ \\
\hline $\mathrm{J} 1106.6+7234$ & 166.654 & 72.571 & $6.45 \pm 0.17$ & 38.0 & NGC 3516 & Sy 1.5 & 0.0088 & $\mathrm{y}$ \\
\hline $\mathrm{J} 1115.9+5426$ & 168.999 & 54.450 & $0.88 \pm 0.15$ & 5.7 & SDSS J111519.98+542316.6 & Sy2 & 0.0703 & \\
\hline $\mathrm{J} 1125.4+5421$ & 171.352 & 54.351 & $0.97 \pm 0.15$ & 6.3 & ARP 151 & Sy1 & 0.0210 & \\
\hline $\mathrm{J} 1127.5+1908$ & 171.900 & 19.148 & $1.12 \pm 0.20$ & 5.6 & 1RXS J112716.6+190914 & Sy1 & 0.1050 & $\mathrm{y}$ \\
\hline $\mathrm{J} 1132.7+5259$ & 173.188 & 52.988 & $1.01 \pm 0.15$ & 6.6 & UGC 6527 & Sy1 & 0.0277 & $\mathrm{y}$ \\
\hline $\mathrm{J} 1136.5+2132$ & 174.150 & 21.548 & $1.13 \pm 0.19$ & 5.9 & Mrk 739 & Sy1 & 0.0299 & \\
\hline J1139.0-3744 & 174.764 & -37.741 & $10.07 \pm 0.27$ & 37.9 & NGC 3783 & Sy1 & 0.0097 & $\mathrm{y}$ \\
\hline $\mathrm{J} 1139.1+5912$ & 174.783 & 59.212 & $1.25 \pm 0.15$ & 8.1 & SBS $1136+594$ & Sy 1.5 & 0.0601 & \\
\hline J1139.4+3156 & 174.869 & 31.935 & $1.00 \pm 0.17$ & 5.8 & NGC 3786 & Sy 1.8 & 0.0089 & \\
\hline $\mathrm{J} 1144.7+7939$ & 176.190 & 79.662 & $2.13 \pm 0.18$ & 11.9 & SWIFT J1143.7+7942 & Sy 1.2 & 0.0153 & $\mathrm{y}$ \\
\hline $\mathrm{J} 1145.3+5859$ & 176.349 & 59.000 & $0.81 \pm 0.15$ & 5.3 & Ark 320 & GALAXY & 0.0099 & \\
\hline $\mathrm{J} 1145.5-1825$ & 176.393 & -18.428 & $2.84 \pm 0.27$ & 10.5 & 2MASX J11454045-1827149 & Sy1 & 0.0329 & $\mathrm{y}$ \\
\hline $\mathrm{J} 1148.9+2938$ & 177.230 & 29.634 & $1.06 \pm 0.18$ & 6.1 & MCG $+05-28-032$ & LINER & 0.0230 & \\
\hline $\mathrm{J} 1158.0+5526$ & 179.502 & 55.449 & $1.04 \pm 0.15$ & 6.9 & NGC 3998 & Seyfert & 0.0036 & $\mathrm{y}$ \\
\hline $\mathrm{J} 1201.0+0647$ & 180.250 & 6.800 & $1.18 \pm 0.21$ & 5.6 & SWIFT J1200.8+0650 & GALAXY & 0.0360 & $\mathrm{y}$ \\
\hline $\mathrm{J} 1203.0+4432$ & 180.773 & 44.534 & $2.33 \pm 0.15$ & 15.1 & NGC 4051 & Sy 1.5 & 0.0023 & $\mathrm{y}$ \\
\hline $\mathrm{J} 1204.5+2018$ & 181.149 & 20.301 & $1.32 \pm 0.19$ & 7.1 & ARK 347 & Sy2 & 0.0225 & $\mathrm{y}$ \\
\hline $\mathrm{J} 1206.2+5242$ & 181.565 & 52.710 & $1.24 \pm 0.15$ & 8.3 & NGC 4102 & GALAXY & 0.0028 & y \\
\hline $\mathrm{J} 1209.1+4700$ & 182.300 & 47.000 & $0.76 \pm 0.15$ & 5.0 & Mrk 198 & Sy2 & 0.0246 & $\mathrm{y}$ \\
\hline J1209.4+4341 & 182.370 & 43.686 & $1.56 \pm 0.15$ & 10.1 & NGC 4138 & Sy1.9 & 0.0030 & $\mathrm{y}$ \\
\hline $\mathrm{J} 1210.5+3924$ & 182.633 & 39.406 & $24.60 \pm 0.16$ & 153.4 & NGC 4151 & Sy 1.5 & 0.0033 & $\mathrm{y}$ \\
\hline $\mathrm{J} 1210.6+3819$ & 182.667 & 38.333 & $0.96 \pm 0.16$ & 6.0 & LEDA 38759 & Sy1 & 0.0230 & \\
\hline $\mathrm{J} 1217.2+0711$ & 184.300 & 7.200 & $1.21 \pm 0.20$ & 5.9 & NGC 4235 & Sy1 & 0.0080 & $\mathrm{y}$ \\
\hline $\mathrm{J} 1218.3+2950$ & 184.593 & 29.839 & $1.53 \pm 0.17$ & 9.1 & Mrk 766 & Sy 1.5 & 0.0127 & $\mathrm{y}$ \\
\hline $\mathrm{J} 1219.0+4715$ & 184.750 & 47.252 & $0.96 \pm 0.15$ & 6.3 & NGC 4258 & LINER & 0.0015 & $\mathrm{y}$ \\
\hline $\mathrm{J} 1222.0+7518$ & 185.503 & 75.311 & $1.27 \pm 0.17$ & 7.4 & Mrk 205 & Sy1 & 0.0700 & $\mathrm{y}$ \\
\hline $\mathrm{J} 1225.7+1239$ & 186.447 & 12.665 & $12.58 \pm 0.19$ & 65.6 & NGC 4388 & Sy2 & 0.0084 & $\mathrm{y}$ \\
\hline
\end{tabular}


Table 5

(Continued)

\begin{tabular}{|c|c|c|c|c|c|c|c|c|}
\hline SWIFT NAME & $\begin{array}{c}\text { R.A. } \\
\text { (J2000) }\end{array}$ & $\begin{array}{c}\text { Decl. } \\
\text { (J2000) }\end{array}$ & $\begin{array}{c}\text { Flux } \\
\left(10^{-11} \mathrm{cgs}\right)\end{array}$ & $\mathrm{S} / \mathrm{N}$ & $\mathrm{ID}^{\mathrm{a}}$ & Type $^{\text {b }}$ & Redshift & In BAT 22 months $^{\mathrm{c}}$ ? \\
\hline $\mathrm{J} 1225.8+3330$ & 186.466 & 33.513 & $1.25 \pm 0.16$ & 7.7 & NGC 4395 & Sy1 & 0.0010 & $\mathrm{y}$ \\
\hline J1235.6-3955 & 188.902 & -39.919 & $10.21 \pm 0.26$ & 39.4 & NGC 4507 & Sy2 & 0.0118 & $\mathrm{y}$ \\
\hline $\mathrm{J} 1238.8-2718$ & 189.723 & -27.308 & $4.39 \pm 0.28$ & 15.9 & ESO 506-027 & Sy2 & 0.0240 & $\mathrm{y}$ \\
\hline $\mathrm{J} 1239.0-1611$ & 189.769 & -16.196 & $2.02 \pm 0.26$ & 7.7 & XSS J12389-1614 & Sy2 & 0.0360 & $\mathrm{y}$ \\
\hline $\mathrm{J} 1239.5-0520$ & 189.898 & -5.341 & $4.52 \pm 0.23$ & 19.8 & NGC 4593 & Sy1 & 0.0090 & $\mathrm{y}$ \\
\hline $\mathrm{J} 1246.6+5434$ & 191.661 & 54.575 & $1.34 \pm 0.15$ & 9.0 & NGC 4686 & GALAXY & 0.0168 & $\mathrm{y}$ \\
\hline $\mathrm{J} 1302.8+1624$ & 195.700 & 16.400 & $0.90 \pm 0.17$ & 5.1 & Mrk 0783 & Sy1.2 & 0.0672 & \\
\hline $\mathrm{J} 1306.7-4024$ & 196.698 & -40.415 & $2.37 \pm 0.27$ & 8.9 & ESO 323-077 & Sy 1.2 & 0.0150 & $\mathrm{y}$ \\
\hline $\mathrm{J} 1309.1+1137$ & 197.279 & 11.632 & $2.19 \pm 0.18$ & 12.0 & SWIFT J1309.2+1139 & GALAXY & 0.0251 & $\mathrm{y}$ \\
\hline $\mathrm{J} 1315.4+4424$ & 198.852 & 44.404 & $1.28 \pm 0.15$ & 8.4 & IGR J13149+4422 & Seyfert & 0.0367 & \\
\hline $\mathrm{J} 1322.3-1642$ & 200.591 & -16.716 & $2.57 \pm 0.27$ & 9.5 & MCG -03-34-064 & Sy 1.8 & 0.0165 & $\mathrm{y}$ \\
\hline $\mathrm{J} 1325.4-4301$ & 201.366 & -43.017 & $49.77 \pm 0.27$ & 187.6 & Cen A & Sy2 & 0.0018 & $\mathrm{y}$ \\
\hline $\mathrm{J} 1334.8-2323$ & 203.700 & -23.400 & $1.49 \pm 0.29$ & 5.1 & ESO 509-38 & Sy2 & 0.0265 & \\
\hline J1335.7-3418 & 203.944 & -34.302 & $4.86 \pm 0.29$ & 16.6 & MCG -06-30-015 & Sy 1.2 & 0.0077 & $\mathrm{y}$ \\
\hline $\mathrm{J} 1338.1+0433$ & 204.547 & 4.552 & $3.63 \pm 0.20$ & 17.9 & NGC 5252 & Sy2 & 0.0230 & $\mathrm{y}$ \\
\hline $\mathrm{J} 1341.4+3022$ & 205.356 & 30.369 & $1.15 \pm 0.16$ & 7.2 & Mrk 268 & Sy2 & 0.0404 & \\
\hline $\mathrm{J} 1349.5-3018$ & 207.390 & -30.304 & $17.87 \pm 0.31$ & 58.2 & IC 4329A & Sy1 & 0.0161 & $\mathrm{y}$ \\
\hline J1353.2+6919 & 208.305 & 69.327 & $2.78 \pm 0.17$ & 16.7 & Mrk 279 & Sy 1.5 & 0.0305 & $\mathrm{y}$ \\
\hline $\mathrm{J} 1356.1+3835$ & 209.033 & 38.583 & $1.22 \pm 0.16$ & 7.7 & Mrk 464 & Sy1 & 0.0507 & $\mathrm{y}$ \\
\hline $\mathrm{J} 1408.4-3024$ & 212.100 & -30.400 & $1.65 \pm 0.32$ & 5.1 & PGC 050427 & Sy1 & 0.0235 & \\
\hline $\mathrm{J} 1413.5-0312$ & 213.375 & -3.201 & $14.38 \pm 0.24$ & 59.0 & NGC 5506 & Sy1.9 & 0.0062 & $\mathrm{y}$ \\
\hline $\mathrm{J} 1418.2+2507$ & 214.568 & 25.133 & $3.12 \pm 0.17$ & 18.2 & NGC 5548 & Sy 1.5 & 0.0172 & $\mathrm{y}$ \\
\hline J1419.5-2639 & 214.893 & -26.663 & $3.49 \pm 0.34$ & 10.4 & ESO 511-G030 & Sy1 & 0.0224 & $\mathrm{y}$ \\
\hline $\mathrm{J} 1421.6+4750$ & 215.420 & 47.838 & $1.03 \pm 0.16$ & 6.4 & QSO B1419+480 & Sy1 & 0.0720 & $\mathrm{y}$ \\
\hline $\mathrm{J} 1424.3+2435$ & 216.100 & 24.600 & $0.89 \pm 0.17$ & 5.1 & NGC 5610 & GALAXY & 0.0169 & \\
\hline $\mathrm{J} 1429.6+0117$ & 217.400 & 1.300 & $1.26 \pm 0.23$ & 5.4 & QSO B1426+015 & Sy1 & 0.0860 & \\
\hline $\mathrm{J} 1436.5+5847$ & 219.149 & 58.798 & $1.37 \pm 0.16$ & 8.3 & QSO J1436+5847 & Sy1 & 0.0312 & $\mathrm{y}$ \\
\hline $\mathrm{J} 1441.2+5330$ & 220.300 & 53.500 & $0.85 \pm 0.16$ & 5.1 & Mrk 477 & Sy2 & 0.0380 & \\
\hline J1442.6-1713 & 220.664 & -17.223 & $4.83 \pm 0.34$ & 14.4 & NGC 5728 & Sy2 & 0.0095 & $\mathrm{y}$ \\
\hline $\mathrm{J} 1453.1+2556$ & 223.282 & 25.936 & $1.29 \pm 0.18$ & 7.0 & RX J1453.1+2554 & Sy1 & 0.0465 & \\
\hline $\mathrm{J} 1504.2+1025$ & 226.073 & 10.417 & $1.51 \pm 0.22$ & 6.8 & Mrk 841 & Sy1 & 0.0364 & $\mathrm{y}$ \\
\hline $\mathrm{J} 1515.4+4201$ & 228.868 & 42.033 & $1.05 \pm 0.18$ & 5.9 & NGC 5899 & Sy2 & 0.0085 & $\mathrm{y}$ \\
\hline $\mathrm{J} 1536.2+5753$ & 234.061 & 57.890 & $1.43 \pm 0.18$ & 7.9 & Mrk 290 & Sy1 & 0.0296 & $\mathrm{y}$ \\
\hline J1548.4-1344 & 237.106 & -13.749 & $2.91 \pm 0.39$ & 7.4 & NGC 5995 & Sy2 & 0.0251 & $\mathrm{y}$ \\
\hline $\mathrm{J} 1554.8+3242$ & 238.700 & 32.700 & $1.04 \pm 0.20$ & 5.2 & 2MASX J15541741+3238381 & Sy1 & 0.0483 & \\
\hline $\mathrm{J} 1618.4+3224$ & 244.600 & 32.400 & $1.07 \pm 0.21$ & 5.1 & $3 \mathrm{C} 332$ & RG & 0.1500 & \\
\hline $\mathrm{J} 1628.3+5147$ & 247.082 & 51.793 & $2.45 \pm 0.20$ & 12.4 & SWIFT J1628.1+5145 & Sy 1.9 & 0.0547 & $\mathrm{y}$ \\
\hline $\mathrm{J} 1653.2+0224$ & 253.319 & 2.404 & $4.20 \pm 0.35$ & 12.1 & NGC 6240 & Sy2 & 0.0245 & $\mathrm{y}$ \\
\hline $\mathrm{J} 1822.1+6421$ & 275.541 & 64.361 & $1.10 \pm 0.21$ & 5.3 & QSO B1821+643 & Sy1 & 0.2970 & $\mathrm{y}$ \\
\hline $\mathrm{J} 1824.2-5620$ & 276.057 & -56.348 & $1.98 \pm 0.29$ & 6.9 & IC 4709 & Sy2 & 0.0169 & $\mathrm{y}$ \\
\hline $\mathrm{J} 1835.1+3240$ & 278.791 & 32.683 & $4.67 \pm 0.21$ & 22.0 & $3 C 382$ & Sy1 & 0.0579 & $\mathrm{y}$ \\
\hline J1837.1-5922 & 279.284 & -59.368 & $1.79 \pm 0.28$ & 6.3 & FAIRALL 49 & Sy2 & 0.0200 & $\mathrm{y}$ \\
\hline $\mathrm{J} 1838.6-6523$ & 279.658 & -65.394 & $6.23 \pm 0.28$ & 22.4 & ESO 103-035 & Sy2 & 0.0133 & $\mathrm{y}$ \\
\hline $\mathrm{J} 1842.4+7946$ & 280.616 & 79.771 & $5.82 \pm 0.19$ & 29.9 & 3C 390.3 & Sy1 & 0.0561 & $\mathrm{y}$ \\
\hline $\mathrm{J} 1845.1-6223$ & 281.297 & -62.399 & $2.52 \pm 0.28$ & 9.0 & ESO $140-43$ & Sy 1 & 0.0141 & $\mathrm{y}$ \\
\hline $\mathrm{J} 1857.3-7827$ & 284.341 & -78.464 & $1.86 \pm 0.26$ & 7.1 & LEDA 140831 & Sy1 & 0.0420 & $\mathrm{y}$ \\
\hline $\mathrm{J} 1921.2-5840$ & 290.323 & -58.677 & $3.54 \pm 0.28$ & 12.6 & ESO $141-55$ & Sy1 & 0.0366 & $\mathrm{y}$ \\
\hline J1942.7-1018 & 295.680 & -10.316 & $4.30 \pm 0.30$ & 14.3 & NGC 6814 & Sy1 & 0.0052 & $\mathrm{y}$ \\
\hline $\mathrm{J} 2009.1-6103$ & 302.289 & -61.064 & $3.03 \pm 0.26$ & 11.5 & SWIFT J2009.0-6103 & Sy1 & 0.0149 & $\mathrm{y}$ \\
\hline $\mathrm{J} 2018.3-5538$ & 304.598 & -55.649 & $1.65 \pm 0.27$ & 6.1 & PKS 2014-55 & RG & 0.0600 & \\
\hline $\mathrm{J} 2042.7+7508$ & 310.685 & 75.136 & $3.23 \pm 0.19$ & 16.9 & $4 C+74.26$ & RG & 0.1040 & $\mathrm{y}$ \\
\hline $\mathrm{J} 2044.1-1043$ & 311.039 & -10.731 & $5.62 \pm 0.29$ & 19.7 & Mrk 509 & Sy1.2 & 0.0344 & $\mathrm{y}$ \\
\hline $\mathrm{J} 2052.0-5703$ & 313.017 & -57.063 & $4.63 \pm 0.25$ & 18.3 & IC 5063 & Sy2 & 0.0113 & $\mathrm{y}$ \\
\hline J2109.1-0939 & 317.300 & -9.652 & $1.58 \pm 0.27$ & 5.9 & 1H 2107-097 & LINER & 0.0265 & \\
\hline $\mathrm{J} 2132.1-3343$ & 323.028 & -33.727 & $2.92 \pm 0.27$ & 10.7 & CTS 109 & Sy1 & 0.0297 & $\mathrm{y}$ \\
\hline $\mathrm{J} 2136.0-6223$ & 324.006 & -62.400 & $2.42 \pm 0.23$ & 10.6 & QSO J2136-6224 & Sy1 & 0.0589 & $\mathrm{y}$ \\
\hline $\mathrm{J} 2138.8+3206$ & 324.713 & 32.115 & $1.29 \pm 0.20$ & 6.3 & LEDA 67084 & Sy1 & 0.0250 & \\
\hline $\mathrm{J} 2200.7+1033$ & 330.199 & 10.565 & $1.76 \pm 0.21$ & 8.6 & SWIFT J2200.9+1032 & Sy 1.9 & 0.0266 & $\mathrm{y}$ \\
\hline $\mathrm{J} 2202.1-3152$ & 330.526 & -31.878 & $8.01 \pm 0.25$ & 31.8 & NGC 7172 & Sy2 & 0.0087 & $\mathrm{y}$ \\
\hline $\mathrm{J} 2204.5+0335$ & 331.149 & 3.600 & $1.33 \pm 0.21$ & 6.3 & IRAS $22017+0319$ & Sy2 & 0.0610 & \\
\hline J2209.5-4709 & 332.387 & -47.166 & $3.02 \pm 0.23$ & 13.4 & NGC 7213 & Sy 1.5 & 0.0277 & $\mathrm{y}$ \\
\hline $\mathrm{J} 2223.8-0207$ & 335.962 & -2.121 & $1.93 \pm 0.22$ & 8.9 & $3 \mathrm{C} 445$ & Sy1 & 0.0564 & $\mathrm{y}$ \\
\hline $\mathrm{J} 2235.8-2603$ & 338.966 & -26.054 & $2.76 \pm 0.24$ & 11.6 & NGC 7314 & Sy1.9 & 0.0048 & $\mathrm{y}$ \\
\hline $\mathrm{J} 2236.1+3357$ & 339.040 & 33.952 & $1.66 \pm 0.19$ & 8.8 & Arp 319 & Sy2 & 0.0225 & $\mathrm{y}$ \\
\hline
\end{tabular}


Table 5

(Continued)

\begin{tabular}{|c|c|c|c|c|c|c|c|c|}
\hline SWIFT NAME & $\begin{array}{c}\text { R.A. } \\
\text { (J2000) }\end{array}$ & $\begin{array}{c}\text { Decl. } \\
(\mathrm{J} 2000)\end{array}$ & $\begin{array}{c}\text { Flux } \\
\left(10^{-11} \mathrm{cgs}\right)\end{array}$ & $\mathrm{S} / \mathrm{N}$ & $\mathrm{ID}^{\mathrm{a}}$ & Type $^{\text {b }}$ & Redshift & In BAT 22 months ${ }^{\mathrm{c}}$ ? \\
\hline $\mathrm{J} 2236.8-1235$ & 339.223 & -12.599 & $1.43 \pm 0.23$ & 6.2 & Mrk 915 & Sy1 & 0.0240 & $\mathrm{y}$ \\
\hline $\mathrm{J} 2245.7+3941$ & 341.449 & 39.695 & $1.71 \pm 0.18$ & 9.2 & $3 \mathrm{C} 452$ & Sy2 & 0.0811 & $\mathrm{y}$ \\
\hline J2254.1-1734 & 343.535 & -17.578 & $5.67 \pm 0.23$ & 24.7 & MR 2251-178 & Sy1 & 0.0640 & $\mathrm{y}$ \\
\hline $\mathrm{J} 2258.9+4053$ & 344.749 & 40.899 & $1.31 \pm 0.18$ & 7.2 & UGC 12282 & Sy1 & 0.0171 & $\mathrm{y}$ \\
\hline $\mathrm{J} 2259.5+2455$ & 344.899 & 24.929 & $1.51 \pm 0.19$ & 8.0 & LEDA 70195 & Sy1 & 0.0338 & $\mathrm{y}$ \\
\hline $\mathrm{J} 2303.2+0853$ & 345.809 & 8.885 & $3.87 \pm 0.20$ & 19.4 & NGC 7469 & Sy1.2 & 0.0163 & $\mathrm{y}$ \\
\hline J2304.7-0841 & 346.194 & -8.686 & $6.09 \pm 0.22$ & 27.9 & Mrk 926 & Sy 1.5 & 0.0469 & $\mathrm{y}$ \\
\hline $\mathrm{J} 2304.7+1217$ & 346.200 & 12.300 & $1.11 \pm 0.20$ & 5.6 & NGC 7479 & Sy2 & 0.0079 & \\
\hline $\mathrm{J} 2318.4-4221$ & 349.614 & -42.360 & $4.09 \pm 0.20$ & 20.7 & NGC 7582 & Sy2 & 0.0053 & $\mathrm{y}$ \\
\hline $\mathrm{J} 2319.0+0014$ & 349.762 & 0.241 & $2.82 \pm 0.21$ & 13.5 & NGC 7603 & Sy 1 & 0.0293 & $\mathrm{y}$ \\
\hline $\mathrm{J} 2326.3+2154$ & 351.600 & 21.900 & $0.97 \pm 0.19$ & 5.1 & RBS 20005 & Sy 1 & 0.1200 & \\
\hline $\mathrm{J} 2342.0+3035$ & 355.500 & 30.600 & $1.08 \pm 0.19$ & 5.7 & UGC 12741 & GALAXY & 0.0174 & $\mathrm{y}$ \\
\hline $\mathrm{J} 2358.7-6052$ & 359.699 & -60.876 & $1.10 \pm 0.19$ & 5.9 & PKS 2356-61 & RG & 0.0963 & \\
\hline
\end{tabular}

Notes.

a Sources with a SWIFT name were identified in the works of Tueller et al. (2008, 2009).

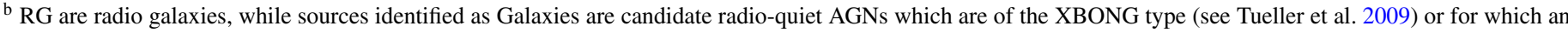
optical spectrum is not yet available.

${ }^{c}$ Is the source detected in the 22 months BAT survey of Tueller et al. (2009)?

\section{REFERENCES}

Abdo, A. A. 2009a, arXiv:0902.1559

Abdo, A. A. 2009b, arXiv:0902.1340

Ahn, K., \& Komatsu, E. 2005, Phys. Rev. D, 72, 061301

Ahn, K., Komatsu, E., \& Höflich, P. 2005, Phys. Rev. D, 71, 121301

Ajello, M., Greiner, J., Kanbach, G., Rau, A., Strong, A. W., \& Kennea, J. A. 2008a, ApJ, 678, 102

Ajello, M., et al. 2008b, ApJ, 689, 666

Ajello, M., et al. 2008c, ApJ, 673, 96

Ajello, M., et al. 2009, ApJ, 690, 367

Antonucci, R. 1993, ARA\&A, 31, 473

Avni, Y. 1976, ApJ, 210, 642

Barnes, J. E., \& Hernquist, L. 1996, ApJ, 471, 115

Barthelmy, S. D., et al. 2005, Space Sci. Rev., 120, 143

Beckmann, V., Engels, D., Bade, N., \& Wucknitz, O. 2003, A\&A, 401, 927

Bell, E. F., McIntosh, D. H., Katz, N., \& Weinberg, M. D. 2003, ApJS, 149, 289

Bhattacharya, D., Sreekumar, P., \& Mukherjee, R. 2009, RAA, 9, 85

Bird, A. J., et al. 2007, ApJS, 170, 175

Blandford, R. D., Netzer, H., Woltjer, L., Courvoisier, T. J.-L., \& Mayor, M. (ed.) 1990, Active Galactic Nuclei (Berlin: Springer)

Blandford, R. D., \& Rees, M. J. 1978, Phys. Scr., 17, 265

Blandford, R. D., \& Znajek, R. L. 1977, MNRAS, 179, 433

Bloemen, H., et al. 1995, A\&A, 293, L1

Bogdanović, T., Reynolds, C. S., \& Miller, M. C. 2007, ApJ, 661, L147

Bongiorno, A., et al. 2007, A\&A, 472, 443

Borgani, S., et al. 2001, ApJ, 561, 13

Bouwens, R. J., Illingworth, G. D., Franx, M., \& Ford, H. 2008, ApJ, 686, 230

Brusa, M., et al. 2009, ApJ, 693, 8

Caccianiga, A., Maccacaro, T., Wolter, A., della Ceca, R., \& Gioia, I. M. 2002, ApJ, 566, 181

Cappelluti, N., et al. 2007, ApJS, 172, 341

Cara, M., \& Lister, M. L. 2008, ApJ, 674, 111

Chiang, J., \& Mukherjee, R. 1998, ApJ, 496, 752

Clayton, D. D., \& Ward, R. A. 1975, ApJ, 198, 241

Comastri, A., Gilli, R., \& Hasinger, G. 2006, arXiv:astro-ph/0604523

Conselice, C. J. 2008, in ASP Conf. Ser. 390, Pathways Through an Eclectic Universe, ed. J. H. Knapen, T. J. Mahoney, \& A. Vazdekis (San Francisco, CA: ASP), 403

Cowie, L. L., Songaila, A., \& Barger, A. J. 1999, AJ, 118, 603

Croton, D. J., et al. 2006, MNRAS, 365, 11

De Lucia, G., Springel, V., White, S. D. M., Croton, D., \& Kauffmann, G. 2006, MNRAS, 366, 499

della Ceca, R., Lamorani, G., Maccacaro, T., Wolter, A., Griffiths, R., Stocke, J. T., \& Setti, G. 1994, ApJ, 430, 533

Dermer, C. D., \& Gehrels, N. 1995, ApJ, 447, 103

Donato, D., Sambruna, R. M., \& Gliozzi, M. 2005, A\&A, 433, 1163

Dotti, M., Colpi, M., Haardt, F., \& Mayer, L. 2007, MNRAS, 379, 956

Dunlop, J. S., \& Peacock, J. A. 1990, MNRAS, 247, 19
Escala, A. 2007, ApJ, 671, 1264

Escala, A., Larson, R. B., Coppi, P. S., \& Mardones, D. 2004, ApJ, 607, 765

Escala, A., Larson, R. B., Coppi, P. S., \& Mardones, D. 2005, ApJ, 630, 152

Falomo, R., Scarpa, R., \& Bersanelli, M. 1994, ApJS, 93, 125

Falomo, R., Scarpa, R., Treves, A., \& Urry, C. M. 2000, ApJ, 542, 731

Fiore, F., et al. 2008, ApJ, 672, 94

Fukada, Y., Hayakawa, S., Kasahara, I., Makino, F., Tanaka, Y., \& Sreekantan, B. V. 1975, Nature, 254, 398

Galbiati, E., et al. 2005, A\&A, 430, 927

Gehrels, N. 1985, Nucl. Instrum. Methods Phys. Res. A, 239, 324

Gehrels, N., et al. 2004, ApJ, 611, 1005

Gendreau, K. C., et al. 1995, PASJ, 47, L5

Georgakakis, A., Hopkins, A. M., Afonso, J., Sullivan, M., Mobasher, B., \& Cram, L. E. 2004, MNRAS, 354, 127

Gilli, R., Comastri, A., \& Hasinger, G. 2007, A\&A, 463, 79

Giommi, P., \& Colafrancesco, S. 2006, arXiv:astro-ph/0602243

Giommi, P., Colafrancesco, S., Cavazzuti, E., Perri, M., \& Pittori, C. 2006, A\&A, 445,843

Giommi, P., \& Padovani, P. 1994, MNRAS, 268, L51

Greiner, J., et al. 2008, Exp. Astron., 23, 91

Grindlay, J. E. 2005, New Astron. Rev., 49, 436

Gruber, D. E., Matteson, J. L., Peterson, L. E., \& Jung, G. V. 1999, ApJ, 520, 124

Hartman, R. C., et al. 1999, ApJS, 123, 79

Hasan, P. 2007, Ap\&SS, 312, 63

Hasinger, G., Miyaji, T., \& Schmidt, M. 2005, A\&A, 441, 417

Healey, S. E., Romani, R. W., Taylor, G. B., Sadler, E. M., Ricci, R., Murphy, T., Ulvestad, J. S., \& Winn, J. N. 2007, ApJS, 171, 61

Healey, S. E., et al. 2008, ApJS, 175, 97

Hogg, D. W. 1999, arXiv:astro-ph/9905116

Hooper, E. J., Impey, C. D., Foltz, C. B., \& Hewett, P. C. 1996, ApJ, 473, 746

Hopkins, A. M., \& Beacom, J. F. 2006, ApJ, 651, 142

Inoue, Y., \& Totani, T. 2008, arXiv:0810.3580

Inoue, Y., Totani, T., \& Ueda, Y. 2008, ApJ, 672, L5

Kauffmann, G., \& Haehnelt, M. 2000, MNRAS, 311, 576

La Franca, F., et al. 2005, ApJ, 635, 864

Lin, Y. C., et al. 1993, ApJ, 416, L53

Madau, P., Ferguson, H. C., Dickinson, M. E., Giavalisco, M., Steidel, C. C., \& Fruchter, A. 1996, MNRAS, 283, 1388

Madejski, G. M., et al. 1995, ApJ, 438, 672

Marcha, M. J. M., Browne, I. W. A., Impey, C. D., \& Smith, P. S. 1996, MNRAS, 281,425

Markwardt, C. B., Tueller, J., Skinner, G. K., Gehrels, N., Barthelmy, S. D., \& Mushotzky, R. F. 2005, ApJ, 633, L77

Marshall, H. L., Tananbaum, H., Avni, Y., \& Zamorani, G. 1983, ApJ, 269, 35

Masetti, N., et al. 2006, A\&A, 455, 11

Massaro, E., Giommi, P., Leto, C., Marchegiani, P., Maselli, A., Perri, M., Piranomonte, S., \& Sclavi, S. 2009, A\&A, 495, 691

Merloni, A. 2004, MNRAS, 353, 1035 
Merloni, A., Heinz, S., \& di Matteo, T. 2003, MNRAS, 345, 1057

Narumoto, T., \& Totani, T. 2006, ApJ, 643, 81

Negroponte, J., \& White, S. D. M. 1983, MNRAS, 205, 1009

O’Dowd, M., Urry, C. M., \& Scarpa, R. 2002, ApJ, 580, 96

Padovani, P., Giommi, P., Landt, H., \& Perlman, E. S. 2007, ApJ, 662, 182

Rector, T. A., Stocke, J. T., Perlman, E. S., Morris, S. L., \& Gioia, I. M. 2000, AJ, 120,1626

Revnivtsev, M., Gilfanov, M., Sunyaev, R., Jahoda, K., \& Markwardt, C. 2003, A\&A, 411, 329

Sambruna, R. M., Tavecchio, F., Ghisellini, G., Donato, D., Holland, S. T., Markwardt, C. B., Tueller, J., \& Mushotzky, R. F. 2007, ApJ, 669, 884

Sambruna, R. M., et al. 2006, ApJ, 646, 23

Sanders, D. B. 2004, Adv. Space Res., 34, 535

Sbarufatti, B., Treves, A., \& Falomo, R. 2005, ApJ, 635, 173

Scheuer, P. A. G., \& Readhead, A. C. S. 1979, Nature, 277, 182

Schmidt, M. 1968, ApJ, 151, 393

Schwope, A., et al. 2000, Astron. Nachr., 321, 1

Shibata, K., Masuda, S., Shimojo, M., Hara, H., Yokoyama, T., Tsuneta, S., Kosugi, T., \& Ogawara, Y. 1995, ApJ, 451, L83

Sikora, M., Błażejowski, M., Moderski, R., \& Madejski, G. M. 2002, ApJ, 577, 78

Sikora, M., Stawarz, Ł., \& Lasota, J.-P. 2007, ApJ, 658, 815

Silverman, J. D., et al. 2008, ApJ, 679, 118

Sowards-Emmerd, D., Romani, R. W., Michelson, P. F., \& Ulvestad, J. S. 2004, ApJ, 609, 564

Springel, V., et al. 2005, Nature, 435, 629

Stanghellini, C., O’Dea, C. P., Dallacasa, D., Baum, S. A., Fanti, R., \& Fanti, C. 1998, A\&AS, 131, 303

Stewart, K. R., Bullock, J. S., Barton, E. J., \& Wechsler, R. H. 2008, arXiv:0811.1218

Stickel, M., Kuehr, H., \& Fried, J. W. 1993, A\&AS, 97, 483

Strigari, L. E., Beacom, J. F., Walker, T. P., \& Zhang, P. 2005, J. Cosmol. Astropart. Phys., JCAP04(2005)17

Tavecchio, F., Maraschi, L., Ghisellini, G., Kataoka, J., Foschini, L., Sambruna, R. M., \& Tagliaferri, G. 2007, ApJ, 665, 980

Toomre, A., \& Toomre, J. 1972, ApJ, 178, 623

Treister, E., \& Urry, C. M. 2005, ApJ, 630, 115
Tueller, J., Mushotzky, R. F., Barthelmy, S., Cannizzo, J. K., Gehrels, N. Markwardt, C. B., Skinner, G. K., \& Winter, L. M. 2008, ApJ, 681, 113

Tueller, J., et al. 2009, arXiv:0903.3037

Ueda, Y., Akiyama, M., Ohta, K., \& Miyaji, T. 2003, ApJ, 598, 886

Urry, C. M., \& Padovani, P. 1995, PASP, 107, 803

Urry, C. M., \& Shafer, R. A. 1984, ApJ, 280, 569

Véron-Cetty, M.-P., \& Véron, P. 2006, A\&A, 455, 773

Voges, W., et al. 1999, A\&A, 349, 389

Volonteri, M., Sikora, M., \& Lasota, J.-P. 2007, ApJ, 667, 704

Wall, J. 2008, arXiv:0807.3792

Wall, J. V., \& Jackson, C. A. 1997, MNRAS, 290, L17

Wall, J. V., Jackson, C. A., Shaver, P. A., Hook, I. M., \& Kellermann, K. I. 2005, A\&A, 434, 133

Wall, J. V., Pope, A., \& Scott, D. 2008, MNRAS, 383, 435

Wang, L., \& Kauffmann, G. 2008, MNRAS, 391, 785

Watanabe, K., Hartmann, D. H., Leising, M. D., \& The, L.-S. 1999, ApJ, 516, 285

Watanabe, K., Hartmann, D. H., Leising, M. D., The, L.-S., Share, G. H., \& Kinzer, R. L. 1997, in AIP Conf. Ser. 410, The Fourth Compton Symp., ed. C. D. Dermer, M. S. Strickman, \& J. D. Kurfess (Melville, NY: AIP), 1223

Watanabe, S., et al. 2009, ApJ, 694, 294

Weidenspointner, G., et al. 2000, in AIP Conf. Ser. 510, The Fifth Compton Symp., ed. M. L. McConnell \& J. M. Ryan (Melville, NY: AIP), 467

Wilks, S. S. 1938, Ann. Math. Stat., 9, 60

Willott, C. J., Rawlings, S., Blundell, K. M., Lacy, M., \& Eales, S. A. 2001, MNRAS, 322, 536

Wilson, A. S., \& Colbert, E. J. M. 1995, ApJ, 438, 62

Winter, L. M., Mushotzky, R. F., Tueller, J., \& Markwardt, C. 2008, ApJ, 674, 686

Wolter, A., \& Celotti, A. 2001, A\&A, 371, 527

Wolter, A., Gioia, I. M., Maccacaro, T., Morris, S. L., \& Stocke, J. T. 1991, ApJ, 369,314

Wyithe, J. S. B., \& Loeb, A. 2003, ApJ, 595, 614

Zdziarski, A. A. 1986, ApJ, 305, 45

Zdziarski, A. A. 1996, MNRAS, 281, L9

Zdziarski, A. A., Poutanen, J., \& Johnson, W. N. 2000, ApJ, 542, 703

Zhang, S., Collmar, W., \& Schönfelder, V. 2005, A\&A, 444, 767

Zickgraf, F.-J., Engels, D., Hagen, H.-J., Reimers, D., \& Voges, W. 2003, A\&A, 406,535 